Cite this: Phys. Chem. Chem. Phys., 2013, 15, 18607

Received 5th June 2013,

Accepted 11th September 2013

DOI: $10.1039 / \mathrm{c} 3 \mathrm{cp} 52344 f$

www.rsc.org/pccp

\title{
Conformational control of benzophenone-sensitized charge transfer in dinucleotides $\uparrow$
}

\author{
Thomas Merz, $\ddagger^{\mathrm{a}}$ Matthias Wenninger,,$^{\mathrm{b}}$ Michael Weinberger, ${ }^{\mathrm{c}}$ Eberhard Riedle, ${ }^{\mathrm{b}}$ \\ Hans-Achim Wagenknecht ${ }^{\star c}$ and Martin Schütz ${ }^{\star a}$
}

\begin{abstract}
Charge transfer in DNA cannot be understood without addressing the complex conformational flexibility, which occurs on a wide range of timescales. In order to reduce this complexity four dinucleotide models $\mathbf{1 X}$ consisting of benzophenone linked by a phosphodiester to one of the natural nucleosides $\mathbf{X}=\mathbf{A}, \mathbf{G}, \mathbf{T}$, $\mathbf{C}$ were studied in water and methanol. The theoretical work focuses on the dynamics and electronic structure of 1G. Predominant conformations in the two solvents were obtained by molecular dynamics simulations. 1G in $\mathrm{MeOH}$ adopts mainly an open geometry with a distance of 12-16 $\AA$ between the two aromatic parts. In $\mathrm{H}_{2} \mathrm{O}$ the two parts of $\mathbf{1 G}$ form primarily a stacked conformation yielding a distance of 5-6 $\AA$. The low-lying excited states were investigated by electronic structure theory in a QM/MM environment for representative snapshots of the trajectories. Photo-induced intramolecular charge transfer in the $S_{1}$ state occurs exclusively in the stacked conformation. Ultrafast transient absorption spectroscopy with $\mathbf{1 X}$ reveals fast charge transfer from $S_{1}$ in both solvents with varying yields. Significant charge transfer from the $T_{1}$ state is only found for the nucleobases with the lowest oxidation potential: in $\mathrm{H}_{2} \mathrm{O}$, charge transfer occurs with $3.2 \times 10^{9} \mathrm{~s}^{-1}$ for $\mathbf{1 A}$ and $6.0 \times 10^{9} \mathrm{~s}^{-1}$ for $\mathbf{1 G}$. The reorganization energy remains nearly unchanged going from $\mathrm{MeOH}$ to the more polar $\mathrm{H}_{2} \mathrm{O}$. The electronic coupling is rather low even for the stacked conformation with $H_{\mathrm{AB}}=3 \mathrm{meV}$ and explains the moderate charge transfer rates. The solvent controls the conformational distribution and therefore gates the charge transfer due to differences in distance and stacking.
\end{abstract}

\section{Introduction}

Charge transfer in DNA is a phenomenon with two opposing "faces". On the one hand, charge transfer has been intensively studied over the last twenty years. ${ }^{1}$ Yet on the other hand it has become obvious that charge transfer processes in DNA cannot be understood without explicitly addressing the manifold of conformational states present in DNA. ${ }^{2}$ The concept of "conformational gating" has been presented to describe the influence of DNA conformations and conformational flexibility on charge transfer. ${ }^{3}$ The main problem is that the conformational flexibility of double

\footnotetext{
${ }^{a}$ Institute of Physical and Theoretical Chemistry, University of Regensburg, Universitätsstraße 31, D-93040 Regensburg, Germany. E-mail: martin.schuetz@chemie.uni-regensburg.de

${ }^{b}$ Lehrstuhl für BioMolekulare Optik, Ludwig-Maximilians-Universität München, Oettingenstraße 67, 80538 Munich, Germany

${ }^{c}$ Karlsruhe Institute of Technology, Department of Chemistry, Fritz-Haber-Weg 6, Campus Süd, Geb. 30.42, 76131 Karlsruhe, Germany

$\dagger$ Electronic supplementary information (ESI) available: Details of (1) preparation of dinucleotides, (2) transient spectroscopic methods and raw data, (3) modeling of the ET dynamics with Marcus theory and (4) computational work. See DOI: $10.1039 / \mathrm{c} 3 \mathrm{cp} 52344 \mathrm{f}$

$\neq$ These authors contributed equally.
}

helical DNA is very complex and occurs on multiple timescales. Since charge transfer rates strongly depend on the DNA base environment we expect not to observe single kinetic rate constants for DNA-mediated charge transfer, but rather a distribution of rates. ${ }^{4}$ Hence, the central question is how the issue of conformational influence on charge transfer can be addressed properly by the combination of time-resolved measurements and theory. For this purpose, we designed the dinucleotides $\mathbf{1 X}$ as the smallest possible models for photoinduced charge transfer in DNA. They consist of benzophenone as an artificial C-nucleoside linked by a phosphodiester bridge to one of the natural nucleosides ( $\mathrm{X}=\mathrm{A}, \mathrm{G}, \mathrm{T}$ or $\mathrm{C}$ ). These dinucleotides are soluble both in water and in polar organic solvents like $\mathrm{MeOH}$. It is expected that the conformational scenario changes significantly between water and $\mathrm{MeOH}$ and, therefore, the conformational influence on photoinduced singlet and triplet charge transfer ${ }^{5}$ can be studied in full detail by both time-resolved transient absorption spectroscopy and theory, including molecular dynamics and quantum chemical calculations to describe the charge transfer states.

Using benzophenone as the photo-induced electron acceptor has the advantage that the dinucleotides $\mathbf{1 X}$ represent both 
interesting biologically relevant systems with respect to DNA damage $^{6}$ and the starting point for future applications in chemical biology and photocatalysis. Benzophenone (BP) is a well characterized organic chromophore for biological photochemistry and chemical photocatalysis due to the very efficient inter-system crossing (ISC) to the triplet $\left(\mathrm{T}_{1}\right)$ state. ${ }^{7-17}$ With respect to photoaffinity labeling there are several other advantages: ${ }^{8}$ (i) benzophenone is chemically more stable than azides and diazirines, especially under the special or harsh conditions of peptide and nucleic acid synthesis. (ii) The $\mathrm{T}_{1}$ state of BP is able to extract hydrogen atoms from unreactive $\mathrm{C}-\mathrm{H}$ bonds in biopolymers, e.g., $\alpha$-hydrogen from amino acids. ${ }^{9}$ (iii) The absorption of BP in the UV-A region allows selective excitation outside the typical absorption range of biopolymers including tryptophan in proteins and DNA/RNA bases. On the other hand, BP plays an increasingly important role as an excitation antenna collecting the light and thereby initiating substrate conversion in photochemical reactions. ${ }^{10,11}$ Molecular $\mathrm{BP}$ architectures can be considered as chemical photocatalysts if they bear a substrate binding site, and substrate conversion occurs initiated by energy or electron transfer. ${ }^{12}$ In fact, both processes have been applied successfully for the development of BP- and xanthone-based photoorganocatalysts. $^{13}$ Template-assisted photocatalysis yields enantioselective $[2+2]$ cycloaddition $^{14-16}$ and, more importantly, templated photoinduced electron transfer can be applied to enantioselective aminocyclizations. ${ }^{17}$ With respect to the mentioned properties of BP and its derivatives, covalent conjugates with nucleosides and nucleic acids could be of significant interest. ${ }^{18-23}$ New photoaffinity labels based on BP-modified nucleic acids could help to identify DNA- and RNA-binding proteins. On the other hand, $\mathrm{BP}$ as an artificial nucleoside provides the molecular basis to develop photocatalytically active DNAzymes. Among the few examples found in the literature, BP-substituted nucleosides have been prepared as models for ribonucleotide reductases ${ }^{18,19}$ and as photoreactive dyads. ${ }^{20} \mathrm{BP}$ has been attached to phosphothioates in $\mathrm{RNA}^{21}$ and to $2^{\prime}$-deoxyuridine in $\mathrm{DNA}^{22}$ to form interstrand crosslinks. 4-Cyanobenzophenone-substituted 2'-deoxyuridines have been extensively used for photoinduced charge transfer studies with DNA. ${ }^{23,24}$

We recently presented the synthesis of a novel C-nucleoside consisting of BP directly attached to the anomeric center of $2^{\prime}$-deoxyribofuranoside. ${ }^{25}$ Thereby, the chromophore is placed as an artificial DNA base and can be incorporated synthetically into oligonucleotides by automated phosphoramidite chemistry. In the present study, we furnish a complete characterization of the optical properties of $\mathbf{1}$ as an artificial nucleoside and in the context of four different dinucleotides (1G, 1A, 1C and 1T, Scheme 1) to evaluate the influence of the neighboring DNA base on the BP properties. The photophysical properties of $\mathbf{1}$ can be influenced by two different protic solvents, water and methanol. Time-resolved measurements of singlet and triplet lifetimes elucidate how conformational changes control the photophysical properties of BP and gate the charge transfer in the dinucleotides.

The theoretical part of our work focuses on the dynamics and electronic structure of the dinucleotide $\mathbf{1 G}$, which is the most interesting dinucleotide based on the knowledge that BP-initiated

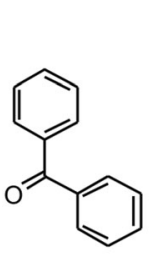

BP

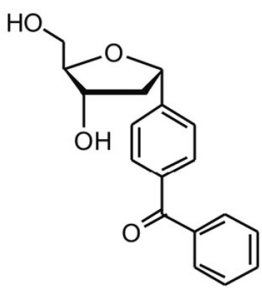

1

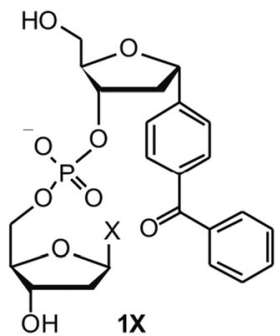

Scheme 1 BP nucleoside 1 and dinucleotides $1 \mathrm{X}$ with $\mathrm{BP}$ as an artificial DNA base $(X=G, T, A, C)$

charge transfer could lead to oxidation of guanine, ${ }^{23,26,27}$ and the results from the time resolved studies (vide infra) that will be described. At first, the isolated 1G (and for comparison 1T) dinucleotides were investigated. Geometry optimizations were performed for the electronic ground state, and excitation energies were computed at the resulting minimum energy geometry. Second, 1G was also investigated in two different solvent environments $\left(\mathrm{H}_{2} \mathrm{O}\right.$ and $\left.\mathrm{MeOH}\right)$ via Molecular Dynamics (MD) simulations and subsequent hybrid Quantum Mechanics/Molecular Mechanics (QM/MM) calculations. Representative snapshots from related MD production trajectories were selected, and the individual geometries reoptimized at the QM/MM level. At the resulting geometries, excitation energies and other properties of the relevant low-lying excited states were calculated in the QM/MM framework. In the following sections we present the detailed results of these calculations.

\section{Theoretical elucidation of conformations and molecular dynamics in water and methanol}

First, DFT geometry optimizations (BP functional, ${ }^{28}$ def-SVP basis $\operatorname{set}^{29}$ ) were performed for the isolated 1G and 1T molecules. For simplicity, 1G and 1T were considered as neutral molecules with a proton added to the phosphate in all calculations presented here. Two strongly differing basic geometries of 1G and 1T were found; (i) a compact, folded structure for 1G as displayed in Fig. 1(b), with the $\mathrm{G}$ and one of the phenyl rings undergoing $\pi$-stacking, and (ii) a stretched, unfolded structure as shown in Fig. 1(a) (analog figures for the folded and unfolded structure of 1T can be found in Fig. SI-19, ESI $\dagger$ ).

The latter constitutes in both cases a local minimum on the potential energy surface. At the Coupled Cluster level (CC2 model) ${ }^{30}$ in the same basis the unfolded structure is not stable and folds to the geometry given in Fig. 1(a). The absence of long-range van der Waals dispersion in the pure DFT/BP description is mainly responsible for the existence of the unfolded structure, which can be repaired to some extent, by e.g. the inclusion of Grimme's-D2 correction, ${ }^{31}$ which is employed in the subsequent DFT QM/MM calculations. Nevertheless, the unfolded structure reappears again if we perform the calculations not in vacuo but in a methanol environment (vide infra). Hence, both folded and unfolded structures were further investigated. 


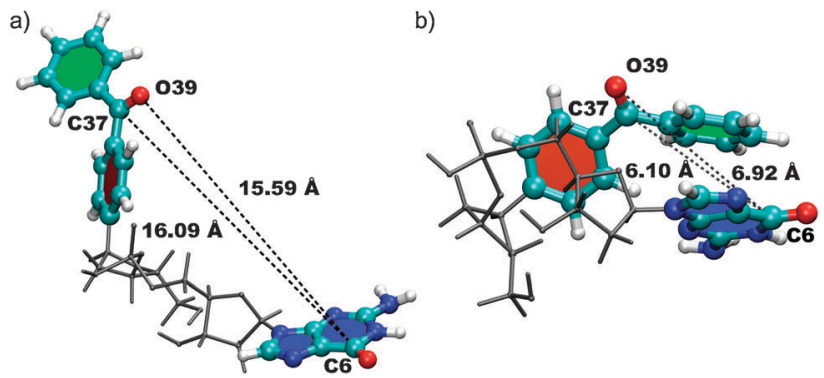

Fig. 1 Optimized structures of $1 \mathbf{G}$ in the gas phase. The five- and six-membered rings are colored in red/green and blue for better recognition. Also the distances $d($ C6-O39) and $d(C 6-C 37)$ are given. (a) Unfolded structure computed with DFT/BP in the def-SVP basis; $d(C 6-039)=15.59 \AA$ and $d(C 6-C 3)=16.09 \AA$. (b) Folded structure computed with CC2/def-SVP; $d(C 6-039)=6.92 \AA$ and $d(C 6-C 37)=6.10 \AA$.

Next, for the isolated dinucleotides, excitation energies, density differences between excited and ground state, and related dipole moment differences of the three lowest singlet states were calculated at the respective ground state minima. To this end, TD-DFT (B3LYP functional ${ }^{32,33}$ ), as well as TD-CC2 response ${ }^{30,34-36}$ (often casually termed as just CC2) were applied. The def-SVP and augcc-pVDZ basis ${ }^{37}$ sets were used for these calculations (for the latter basis set excitation energies were computed only). The resulting excitation energies of the three lowest excited singlet states are compiled in Table 1 for the unfolded and folded geometries of $\mathbf{1 G}$ and $\mathbf{1 T}$ (triplet excitation energies for $\mathbf{1 G}$ can be found in the ESI, $\uparrow c f$. Table SI-2, for comparison).

Fig. 2 depicts the related electron density differences for the case of the folded geometry of $\mathbf{1 G}$ (related electron density differences of the folded geometry of $\mathbf{1 T}$ are displayed in Fig. SI-20 (ESI + ); for the relevant molecular orbitals for 1G and 1T $c f$. Fig. SI-21 and SI-22 (ESI $\dagger$ )).

For the folded geometry of $\mathbf{1 G}$ the $\mathrm{S}_{1}, \mathrm{~S}_{2}$, and $\mathrm{S}_{3}$ states are of $\left(\mathrm{n} \pi^{*}\right)$, charge transfer (CT), and $\left(\pi \pi^{*}\right)$ type. The CT state features depletion of electron density on G, and increase of electron density on the BP part. Comparison of the TD-DFT vs. TD-CC2 results shows a substantial increase in the energy gap between the $\mathrm{S}_{1}$ and $\mathrm{S}_{2}$ from $0.18 \mathrm{eV}$ (TD-DFT) to $0.85 \mathrm{eV}$ (TD-CC2, def SVP) and $0.70 \mathrm{eV}$ (TD-CC2, aug-cc-PVDZ). CT states are notoriously underestimated by TD-DFT with local, or semi-local functionals

Table 1 Results for folded and unfolded molecules of 1G and 1T; optimized ground state; TD-B3LYP/def-SVP TD-CC2/def-SVP and TD-CC2/aug-cc-pVDZ

\begin{tabular}{|c|c|c|c|c|c|c|c|c|c|}
\hline \multirow[b]{3}{*}{ Method } & & \multicolumn{4}{|c|}{ Folded geometry } & \multicolumn{4}{|c|}{ Unfolded geometry } \\
\hline & & \multicolumn{2}{|l|}{$1 \mathrm{G}$} & \multicolumn{2}{|l|}{$1 \mathrm{~T}$} & \multicolumn{2}{|l|}{$1 \mathrm{G}$} & \multicolumn{2}{|l|}{$1 \mathrm{~T}$} \\
\hline & & Type & $\omega[\mathrm{eV}]$ & Type & $\omega[\mathrm{eV}]$ & Type & $\omega[\mathrm{eV}]$ & Type & $\omega[\mathrm{eV}]$ \\
\hline \multirow[t]{3}{*}{ TD-B3LYP } & $\mathrm{S}_{1}$ & $\left(\mathrm{n} \pi^{*}\right)$ & 3.36 & $\left(\mathrm{n} \pi^{*}\right)$ & 3.53 & $\left(\mathrm{n} \pi^{*}\right)$ & 3.50 & $\left(\mathrm{n} \pi^{*}\right)$ & 3.50 \\
\hline & $\mathrm{S}_{2}$ & CT & 3.54 & $\mathrm{CT}$ & 4.16 & CT & 3.56 & CT & 4.42 \\
\hline & $\mathrm{S}_{3}$ & $\left(\pi \pi^{*}\right)$ & 4.42 & $\left(\pi \pi^{*}\right)$ & 4.46 & $\left(\pi \pi^{*}\right)$ & 4.45 & $\left(\pi \pi^{*}\right)$ & 4.46 \\
\hline TD-CC2 & $\mathrm{S}_{1}$ & $\left(\mathrm{n} \pi^{*}\right)$ & 3.74 & $\left(\mathrm{n} \pi^{*}\right)$ & 3.74 & $\left(\mathrm{n} \pi^{*}\right)$ & 3.82 & $\left(\mathrm{n} \pi^{*}\right)$ & 3.76 \\
\hline \multirow[t]{2}{*}{ def-SVP } & $\mathrm{S}_{2}$ & CT & 4.59 & $\left(\pi \pi^{*}\right)$ & 4.79 & $\left(\pi \pi^{*}\right)$ & 4.88 & $\left(\pi \pi^{*}\right)$ & 4.82 \\
\hline & $\mathrm{S}_{3}$ & $\left(\pi \pi^{*}\right)$ & 4.78 & $\left(\pi \pi^{*}\right)$ & 4.83 & $\left(\pi \pi^{*}\right)$ & 4.96 & $\left(\pi \pi^{*}\right)$ & 4.90 \\
\hline TD-CC2 & $\mathrm{S}_{1}$ & $\left(\mathrm{n} \pi^{*}\right)$ & 3.63 & $\left(\mathrm{n} \pi^{*}\right)$ & 3.61 & $\left(\mathrm{n} \pi^{*}\right)$ & 3.63 & $\left(\mathrm{n} \pi^{*}\right)$ & 3.63 \\
\hline \multirow[t]{2}{*}{$\mathrm{a}-\mathrm{VDZ}$} & $\mathrm{S}_{2}$ & CT & 4.33 & $\left(\pi \pi^{*}\right)$ & 4.65 & $\left(\pi \pi^{*}\right)$ & 4.69 & $\left(\pi \pi^{*}\right)$ & 4.69 \\
\hline & $\mathrm{S}_{3}$ & $\left(\pi \pi^{*}\right)$ & 4.66 & $\left(\pi \pi^{*}\right)$ & 4.68 & $\left(\pi \pi^{*}\right)$ & 4.78 & $\left(\pi \pi^{*}\right)$ & 4.76 \\
\hline
\end{tabular}

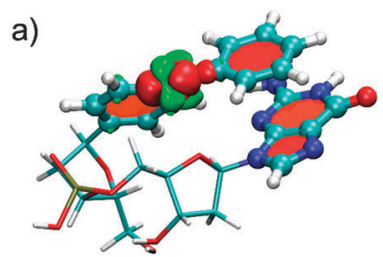

a)
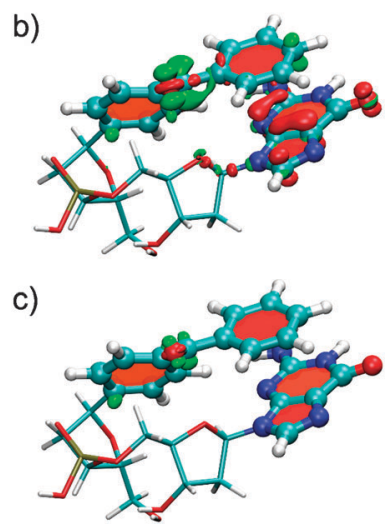

Fig. 2 Electron density difference plots for the first three excited states of the folded geometry of 1G, calculated with TD-CC2 in def-SVP basis. Isosurfaces are plotted for \pm 0.005 a.u., red refers to a decrease, green to an increase in the density upon excitation. (a) $\mathrm{S}_{1}:\left(n \pi^{\star}\right)$ state; $|\Delta \mu|=1.72 \mathrm{D}$ (b) $\mathrm{S}_{2}$ : CT state; $|\Delta \mu|=$ $10.58 \mathrm{D}$ (c) $\mathrm{S}_{3}:\left(\pi \pi^{\star}\right)$ type state; $|\Delta \mu|=1.32 \mathrm{D}$.

due to the electronic self-interaction problem inherent in DFT. ${ }^{38,39}$ For isolated folded 1T, on the other hand, only the TD-DFT calculation features a CT state among the three lowest states. The order and type of characters are similar to 1G. However, the gap between the $\mathrm{S}_{1}$ and the CT states is significantly increased, compared to $1 \mathrm{G}(0.63$ vs. $0.18 \mathrm{eV})$. For TD-CC2, the CT state disappears among the three lowest excitations, and the $S_{1}, S_{2}$ and $S_{3}$ states correspond to a $\left(n \pi^{*}\right),\left(\pi \pi^{*}\right)$ and $\left(\pi \pi^{*}\right)$ types, respectively. Hence, the CT state in the TD-DFT calculation for 1T is just an artifact, caused by the abovementioned electronic self-interaction problem. Adding diffuse functions has the usual effect of decreasing the TD-CC2 excitation energies by $0.1-0.2 \mathrm{eV}$, which is seen in both cases.

For the unfolded geometry the $\mathrm{S}_{1}, \mathrm{~S}_{2}$, and $\mathrm{S}_{3}$ states calculated by TD-CC2 are of $\left(n \pi^{*}\right),\left(\pi \pi^{*}\right)$, and $\left(\pi \pi^{*}\right)$ type for both $1 \mathbf{G}$ and $\mathbf{1 T}$. The CT state for 1G is clearly absent, which is not too surprising since the distance between donor (G) and acceptor (BP) is much larger than in the folded geometry. For TD-DFT, on the other hand, the CT state is clearly present (as the $\mathrm{S}_{2}$ state) and features about the same excitation energy as in the folded geometry. This is the typical irregular behavior of TD-DFT, since CT states described by that method do not reflect the proper distance dependence between donor and acceptor (according to the Coulomb law).

Based on the gas phase results presented so far we conclude that (i) the distance between the BP and the G subsystems intimately affects the character of the three relevant, lowest excited states. In particular there is a low-lying CT state present for the folded geometry, which is absent for the unfolded one. (ii) All these excitations are either localized on G, or BP, or both for the CT state, as is evident from Fig. 2. The sugar and phosphate 
groups linking these two subunits do not play a role and can therefore be safely omitted from the QM part in the subsequent $\mathrm{QM} / \mathrm{MM}$ studies of the system in solvent environments. (iii) A CT state in 1 T cannot be observed, neither in the unfolded, nor the folded geometry.

In order to generate proper starting structures for a subsequent $\mathrm{QM} / \mathrm{MM}$ treatment of $\mathbf{1 G}$ in water and methanol solvent environments, classical molecular dynamics (MD) simulations were carried out first. Similar MD simulations were also carried out for 1T, which however will not be further discussed. For the latter we refer to Fig. SI-26 and SI-27 in the ESI $\dagger$ (Section 4). Also the technical details of these simulations are described in the ESI $†$ (Section 4). MD simulations in water and methanol were performed by starting from either the folded or the unfolded gas phase structure of $\mathbf{1 G}$ (overall four MD simulations). It turns out that the resulting trajectories are rather independent of the starting geometry: 1G solvated in water primarily exists in the folded form, while $\mathbf{1 G}$ solvated in methanol primarily exists in the unfolded form.

The trajectories of the MD simulation of $\mathbf{1 G}$ in methanol (starting from the folded geometry) and of the MD simulation of 1G in water (starting from the unfolded geometry) are displayed in Fig. 3(a) and (b). The trajectories of the two other MD simulations with converse starting geometries are included in Fig. SI-25 (ESI $\dagger$ ). The degree of folding is measured by the distances $d$ (C6-C37) and $d$ (C6-O39) ( $c f$. Fig. SI-18, ESI $\dagger$ ), which reflect the spatial separation of the BP and $\mathrm{G}$ subunits. For the isolated molecule these distances amount to 5-6 A for the folded, and 15-16 ̊̊ for the unfolded form. Thus, in our MD simulations we consider

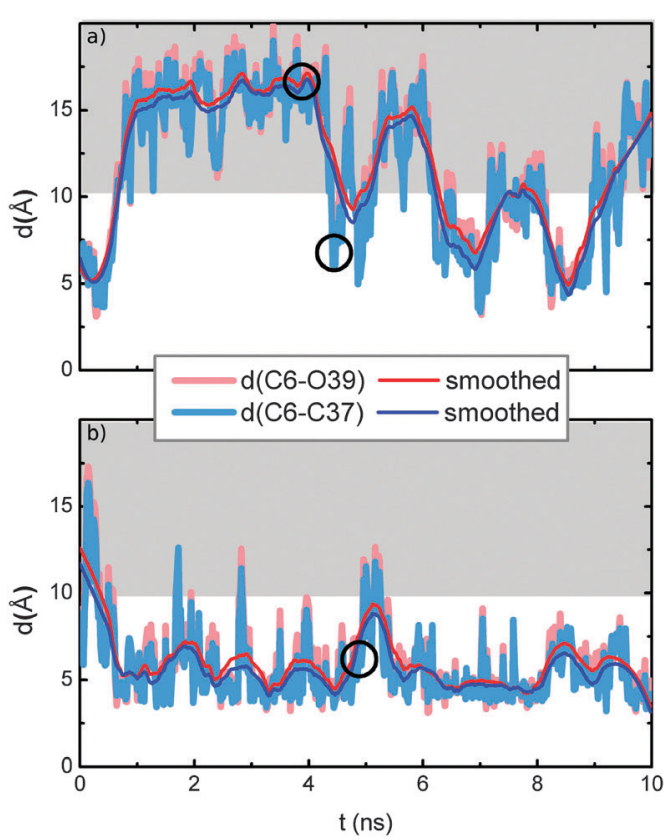

Fig. 3 Trajectories of MD run; (a) shows $1 \mathrm{G}$ in methanol environment and (b) $1 \mathrm{G}$ in water; the distances $d(\mathrm{C} 6-\mathrm{C} 37)$ and $d(\mathrm{C} 6-039)$ in $\AA$ are plotted against the simulation time in ns. The grey area is the region in which the molecule is unfolded. The cycles depict the chosen snapshots. (a) MD of $1 \mathrm{G} \mathrm{MeOH}$, folded (4.2 $\left.\mathrm{ns} ;{ }^{\mathrm{C}} \mathbf{1 G}_{\mathrm{MeOH}}\right)$ and unfolded $\left(4.0 \mathrm{~ns} ;{ }^{\mathrm{a}} \mathbf{1} \mathrm{G}_{\mathrm{MeOH}}\right)$ starting geometry. (b) MD of $\mathbf{1 G}$ water, folded (4.5 ns; ${ }^{\mathrm{a}} \mathbf{1 G}_{\mathrm{H}_{2} \mathrm{O}}$ ) starting geometry. geometries with distances of above $10 \AA$ as unfolded (grey shaded area in Fig. 3), and geometries with distances of below $10 \AA$ as folded forms. Evidently, unfolded 1G in water folds and stays folded most of the time. In contrast, folded 1G in methanol unfolds and stays in that form most of the time. Obviously, in the polar water solvent, the two hydrophobic ring systems BP and G stick together and undergo $\pi$-stacking, while in the less polar methanol they are individually solvated by methanol molecules.

For the subsequent QM/MM study we have selected five representative snapshots: from each of the four trajectories, as indicated in Fig. 3 and in Fig. SI-25 (ESI $\dagger$ ), there are two snapshots related to the water environment, ${ }^{\mathrm{a}} \mathbf{1 G}_{\mathrm{H}_{2} \mathrm{O}}$ and ${ }^{\mathrm{b}} \mathbf{1 G}_{\mathrm{H}_{2} \mathrm{O}}$, corresponding to the folded form, and two related to the methanol environment, ${ }^{\mathrm{a}} \mathbf{1} \mathbf{G}_{\mathrm{MeOH}}$, and ${ }^{\mathrm{b}} \mathbf{1 G}_{\mathrm{MeOH}}$, corresponding to the unfolded form; additionally, a fifth snapshot ${ }^{\mathrm{c}} \mathbf{1 G}_{\mathrm{MeOH}}$, which represents the rather rare event of a folded form in the methanol environment, was also considered.

\section{Spectroscopic results}

For the time resolved measurements and their interpretation the optical properties of the artificial nucleoside $\mathbf{1}$ and the dinucleotides $\mathbf{1 X}$ were characterized. The spectra of compound $\mathbf{1}$ in $\mathrm{MeOH}$ (Fig. 4a and b) are typical for all other compounds. One prominent strong absorption band is centered at $259 \mathrm{~nm}$ $(4.79 \mathrm{eV})$ that corresponds to a ${ }^{1}\left(\pi \pi^{*}\right)$ transition located on BP. A much weaker band is centered at $329 \mathrm{~nm}(3.77 \mathrm{eV})$ and corresponds to the ${ }^{1}\left(\mathrm{n} \pi^{*}\right)$ transition of $\mathrm{BP} .{ }^{40}$ The assignment of the bands to transitions located on $\mathrm{BP}$ is corroborated by the high similarity to the spectrum of BP (Fig. 4a). Furthermore, the band positions are in good agreement with the TD-CC2 excitation energies
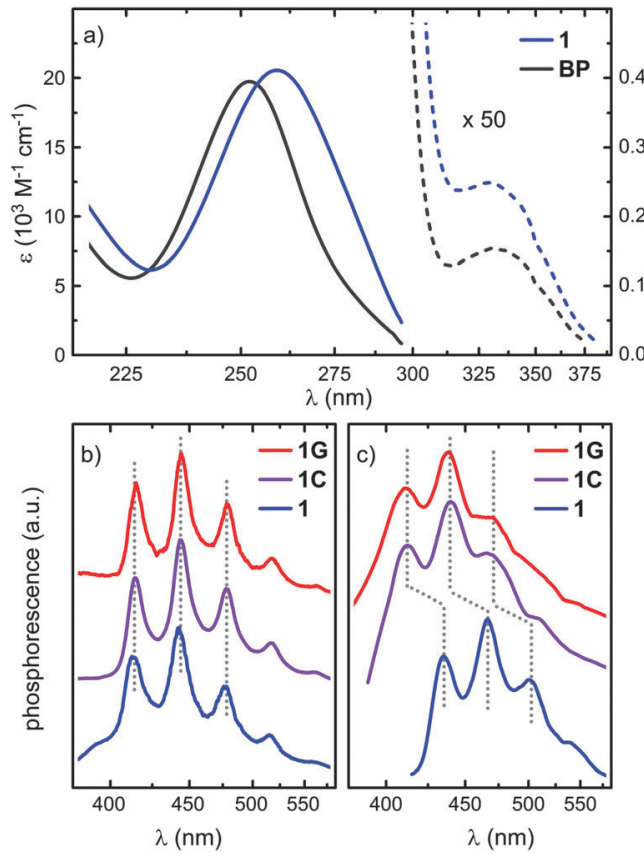

Fig. 4 (a) Absorption of $\mathrm{BP}$ and $\mathbf{1}$ in $\mathrm{MeOH}$ at r.t. (b) Phosphorescence of 1, 1C and $1 \mathrm{G}$ at $77 \mathrm{~K}$ in $\mathrm{MeOH}$. (c) Phosphorescence of $1,1 \mathrm{C}$ and $1 \mathrm{G}$ at $77 \mathrm{~K}$ in $\mathrm{H}_{2} \mathrm{O}$. 
reported in Table 1 . The latter transition allows selective excitation of the BP chromophore outside the nucleic acid absorption range. This is an important prerequisite for the photochemical and photobiological applications. Both electronic transitions are broad and structureless at r.t. due to the thermal population of low frequency vibrational levels and the interaction with the environment.

Upon excitation at $355 \mathrm{~nm}$, nucleoside 1 in $\mathrm{MeOH}$ shows a well structured phosphorescence at $77 \mathrm{~K}$. This structure becomes visible due to the lack of thermal vibrational excitation and the long lifetime of the $\mathrm{T}_{1}$ state. The first vibronic peak corresponding to the $0-0$ vibrationless electronic transition is found at $413 \mathrm{~nm}$. The shape and the energetic positions are very similar for all dinucleotides $\mathbf{1 X}$ (compare spectra for $\mathbf{1 G}$ and $1 \mathrm{C}$ in Fig. 4b). This observation differs from the phosphorescence in aqueous buffer solution (Fig. 4c). 1 dissolved in $\mathrm{H}_{2} \mathrm{O}$ exhibits the $0-0$ peak at $435 \mathrm{~nm}$. Due to the characteristic intensity pattern we can be sure that we observe a shift and not a disappearance of the $0-0$ subband. The shift compared to the $\mathrm{MeOH}$ solution is interpreted as the exposure of the chromophore to water. The $0-0$ phosphorescence peak of the dinucleotides in $\mathrm{H}_{2} \mathrm{O}$ is found to be very similar to $\mathrm{MeOH}$. This shows that the DNA base adjacent to the BP in the dinucleotides is able to shield the chromophore from the electrostatic influence of the water molecules. We therefore expect that the photochemical reactivity of the BP derivative $\mathbf{1}$ as part of the synthesized dinucleotides $\mathbf{1 X}$ is more similar to $\mathbf{1}$ in organic solvents (like $\mathrm{MeOH})$ than $\mathbf{1}$ in water. We can confirm the presence of a preferred stacked (folded) conformation of $\mathbf{1 X}$ in water as suggested by the molecular dynamics simulations.

Electron transfer (ET) between nucleobases and an excited organic chromophore is well established and usually occurs on time scales of 10-100 ps when the two moieties are in close proximity. ${ }^{41,42}$ We used femtosecond transient absorption (TA) spectroscopy ${ }^{43}$ to measure the kinetics of the various compounds. Directly after excitation of the lowest ${ }^{1}\left(n \pi^{*}\right)$ transition, pure BP in $\mathrm{MeOH}$ shows the spectrum of the $\mathrm{S}_{1}$ state with prominent absorption maxima at 340 and $580 \mathrm{~nm}$ (Fig. SI-9, ESI $\dagger$ ). BP in $\mathrm{MeOH}$ serves as reference according to the results obtained with the steady state spectroscopy. The decay of the singlet spectral signatures is dominated by very fast ISC. ${ }^{40}$ Upcoming absorption bands at 320 and $530 \mathrm{~nm}$ can be uniquely assigned to the BP triplet state $\mathrm{T}_{1}$. We find a lifetime of $\tau_{\mathrm{S}_{1}}=12.5 \mathrm{ps}$ for the $\mathrm{S}_{1}$ state. Assuming a quantum yield of $\Phi_{\mathrm{T}}=100 \%$ for the population of the $\mathrm{BP}_{1}$ state in agreement with the literature ${ }^{44}$ the rate of ISC is determined as $k_{\mathrm{ISC}}=\left(\tau_{\mathrm{S}_{1}}\right)^{-1}$. An analogous modeling will also be used in the analysis of the more complex TA data of the BP dinucleotides $\mathbf{1 X}$.

A first measurement series of the BP nucleosides $\mathbf{1 X}$ and reference compound 1 was performed in $\mathrm{MeOH}$ (Fig. 5 and Fig. SI-10 to SI-13, ESI $\dagger$ ). Directly after excitation we observe transient spectra which are very similar to those of pure BP showing the absorption features of the BP $\mathrm{S}_{1}$ state. The covalently bound 2 '-deoxyribofuranoside does not significantly affect the excited state spectral features. The ultrafast ISC is also found for the nucleosides $\mathbf{1 X}$ and reference $\mathbf{1}$, the lifetime of $\mathbf{1}$ in $\mathrm{MeOH}$ is $\tau_{\mathrm{S}_{1}}=9.4 \mathrm{ps}$.

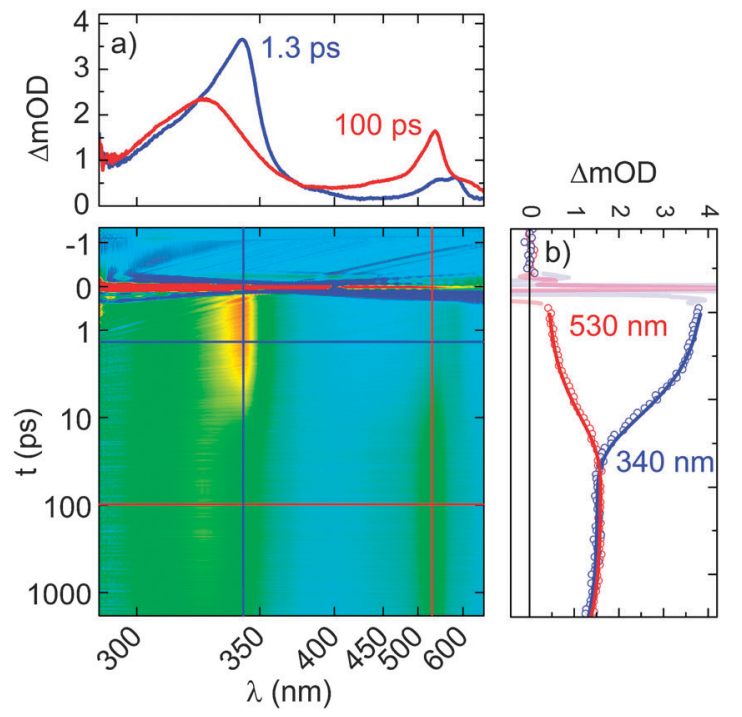

Fig. 5 Transient absorption spectra of $1 \mathrm{C}$ in $\mathrm{MeOH}$ after $358 \mathrm{~nm}$ excitation (a) selected spectra (b) temporal evolution at selected wavelengths (dots) with corresponding traces from the global fit (lines).

The slightly faster ISC compared to BP is reasonable in view of the covalently bound substituent. In comparison to $\mathbf{1}$ all nucleosides 1X show a further reduced lifetime of the $S_{1}$ state and a dependence on the adjacent nucleobase. Especially the lifetime of compound 1G, where BP is linked to guanine (the nucleobase with the lowest standard oxidation potential $E^{0}$ ) is considerably reduced: we find $\tau_{\mathrm{S}_{1}}=7.6 \mathrm{ps}$ in $\mathrm{MeOH}$. All obtained time constants are summarized in Table 2. The lifetime of the triplet states in $\mathrm{MeOH}$ cannot be determined by the ultrafast TA spectroscopy, since it exceeds the maximum pump-probe delay of 2 ns. We therefore set it to infinity for the analysis.

It is interesting to compare the variation of the $S_{1}$ lifetimes $\tau_{\mathrm{S}_{1}}$ with the variation of the standard oxidation potential $E^{0}$ of the corresponding nucleobase. ${ }^{45}$ In 1G, which contains the nucleobase with the lowest $E^{0}$ and hence is the most easily oxidized, $\tau_{\mathrm{S}_{1}}$ is significantly reduced in comparison to all other compounds. This indicates that ET from the covalently bound nucleobase to the excited singlet state of BP can compete with the ISC process, which explains the reduced $\tau_{\mathrm{S}_{1}}$ of all $\mathbf{1 X}$ in comparison to $\mathbf{1}$. The transient signature of the intermediate BP radical anion expected at 333 and $720 \mathrm{~nm}^{46}$ is not visible. A charge recombination process, which is fast in comparison to the ET process, can readily explain the absence of the spectral signatures of the intermediate charge separated state.

From the inhomogeneous distribution of conformations it could be expected that the $S_{1}$ decay time does not conform to a simple exponential behavior. ${ }^{47}$ We fitted the curves with a stretched exponential and obtained somewhat unexpected exponents around 1.1 and not the expected values below 1. A rational can be that we have a highly nonstatistical distribution as we find indeed from the trajectories in Fig. 3. Since this precludes any reasonable analytical modeling, we decided to use the single-exponential fit values for further interpretation.

The ET time constant ${ }^{1} \tau_{\mathrm{ET}}$ determined from the measured lifetime $\tau_{\mathrm{S}_{1}}$ for all dinucleotides $\mathbf{1 X}$ is depicted in Table 2 . 
Table 2 Time constants of excited state dynamics in $\mathrm{MeOH}$ and $\mathrm{H}_{2} \mathrm{O}$ : standard oxidation potential $E^{0}$ of the nucleobase from ref. 45 , $\tau_{\mathrm{s}_{1}}$ obtained from a global fit, ${ }^{1} \tau_{\mathrm{ET}}$ was calculated by using the $\mathrm{S}_{1}$ lifetime of compound 1 in $\mathrm{MeOH}$ as intrinsic lifetime of the $\mathrm{S}_{1}$ state and ${ }^{3} \tau_{\mathrm{ET}}$ from the dynamics of the integrated transient absorption at 300-400 nm. The triplet yield $\Phi_{\mathrm{T}_{1}}$ was determined by inserting the quantities into a rate model based on eqn (3) $\left(\Phi_{\mathrm{T}_{1}}\right.$ theor.) and also experimentally by integration of the triplet specific transient absorption $\left(\Phi_{\mathrm{T}_{1}}\right.$ exp.)

\begin{tabular}{|c|c|c|c|c|c|c|c|c|c|c|}
\hline \multirow[b]{2}{*}{ Sample } & \multirow[b]{2}{*}{$E^{0}[\mathrm{~V}]$} & \multicolumn{5}{|l|}{$\mathrm{MeOH}$} & \multicolumn{4}{|l|}{$\underline{\mathrm{H}_{2} \mathrm{O}}$} \\
\hline & & $\tau_{\mathrm{S}_{1}}[\mathrm{ps}]$ & ${ }^{1} \tau_{\mathrm{ET}}[\mathrm{ps}]$ & ${ }^{1} \Phi_{1}$ theo. & ${ }^{1} \Phi_{1}$ exp. & ${ }^{3} \tau_{\mathrm{ET}}[\mathrm{ns}]$ & $\tau_{\mathrm{S}_{1}}[\mathrm{ps}]$ & ${ }^{1} \tau_{\mathrm{ET}}[\mathrm{ps}]$ & ${ }^{1} \Phi_{1}$ theo. & ${ }^{3} \tau_{\mathrm{ET}}[\mathrm{ns}]$ \\
\hline BP & - & $12.5 \pm 0.5$ & - & - & - & & & & - & \\
\hline $1 \mathrm{C}$ & 2.14 & $8.8 \pm 0.5$ & $140 \pm 50$ & 0.94 & 0.92 & $>2$ & $9.0 \pm 0.8$ & $210 \pm 70$ & 0.96 & $>2$ \\
\hline $1 \mathrm{~T}$ & 2.11 & $9.1 \pm 0.5$ & $290 \pm 100$ & 0.97 & 0.97 & $>2$ & $8.4 \pm 0.8$ & $80 \pm 25$ & 0.89 & $>2$ \\
\hline $\mathbf{1 A}$ & 1.96 & $8.9 \pm 0.5$ & $170 \pm 50$ & 0.95 & 0.89 & $>2$ & $8.2 \pm 0.8$ & $65 \pm 15$ & 0.87 & $0.310 \pm 0.040$ \\
\hline 1G & 1.49 & $7.6 \pm 0.5$ & $40 \pm 10$ & 0.81 & 0.87 & $\sim 1$ & $4.6 \pm 0.5$ & $9 \pm 2$ & 0.49 & $0.166 \pm 0.020$ \\
\hline
\end{tabular}

The ET time constant ${ }^{1} \tau_{\mathrm{ET}}$ was calculated using $k_{\mathrm{ISC}}$ from compound 1 as the intrinsic lifetime of the $S_{1}$ state of BP in the dinucleotide environment as

$$
\left({ }^{1} \tau_{\mathrm{ET}}\right)^{-1}={ }^{1} k_{\mathrm{ET}}=\frac{1}{\tau_{\mathrm{S}_{1}}}-k_{\mathrm{ISC}}
$$

We interpret the dependence of the ET rate on the standard oxidation potential $E^{0}$ of the nucleobase with the help of standard Marcus theory. ${ }^{48}$ For details see the ESI $\dagger$ (Section 3). We calculate $\Delta G^{0}$, which serves as the driving force for the ET reaction between the nucleobase and the $\mathrm{BP} \mathrm{S}_{1}$ or $\mathrm{T}_{1}$ according to Rehm-Weller: ${ }^{49}$

$$
\Delta G^{0}=e\left[E^{0}\left(D^{+\bullet} / D\right)-E^{0}\left(A / A^{-\bullet}\right)\right]-\frac{e^{2}}{4 \pi \varepsilon_{0} \varepsilon r}-\Delta E_{00}
$$

where $E^{0}$ is the one-electron oxidation/reduction potential, $\varepsilon$ is the permittivity of the solvent, $r$ is the average donor-acceptor distance, and $\Delta E_{00}$ is the energy of the excited state relative to the ground state. For the lowest $\mathrm{BP}^{1}\left(\mathrm{n} \pi^{*}\right)$ singlet state, ${ }^{1} \Delta E_{00}$ equals $318 \mathrm{~kJ} \mathrm{~mol}^{-1}(3.30 \mathrm{eV}, 376 \mathrm{~nm})$ and for the lowest triplet state ${ }^{3} \Delta E_{00}$ is $293 \mathrm{~kJ} \mathrm{~mol}^{-1}(3.04 \mathrm{eV}){ }^{49}$ The standard potential $E^{0}$ for the reduction of BP is $E^{0}=-1.00 \mathrm{~V}$ against the normal hydrogen electrode (NHE).$^{50}$ The average distance $r$ between the donor and acceptor was found from the molecular dynamics calculations to be ${ }^{1} r_{\mathrm{MeOH}}={ }^{3} r_{\mathrm{MeOH}}=12 \AA$ for the $\mathrm{S}_{1}$ and $\mathrm{T}_{1}$ states of all $1 \mathrm{X}$ in $\mathrm{MeOH}$, and ${ }^{1} r_{\mathrm{H}_{2} \mathrm{O}}={ }^{3} r_{\mathrm{H}_{2} \mathrm{O}}=6 \AA$ in $\mathrm{H}_{2} \mathrm{O}$. The use of an average value instead of the distance distribution is justified since the distance dependence in eqn (2) only causes minor variations of $\Delta G^{0}$. The values of the permittivity of the solvents are $\varepsilon_{\mathrm{MeOH}}=32.66 \cdot \varepsilon_{0}$ and $\varepsilon_{\mathrm{H}_{2} \mathrm{O}}=78.36 \cdot \varepsilon_{0} \cdot{ }^{51}$ The results of the calculated $\Delta G^{0}$ values are listed in Table 3 for BP in its $\mathrm{S}_{1}$ and $\mathrm{T}_{1}$ states in $\mathrm{MeOH}$ and in $\mathrm{H}_{2} \mathrm{O}$.

Table 3 Change of free energy $\Delta G^{0}$ corresponding to the oxidation of DNA

\begin{tabular}{|c|c|c|c|c|c|}
\hline \multirow[b]{2}{*}{ Sample } & \multirow[b]{2}{*}{$\begin{array}{l}E^{044} \\
{[\mathrm{~V}]}\end{array}$} & \multicolumn{2}{|l|}{$\mathrm{MeOH}$} & \multicolumn{2}{|l|}{$\mathrm{H}_{2} \mathrm{O}$} \\
\hline & & $\begin{array}{l}{ }^{1} \Delta G^{0} \\
{\left[\mathrm{~kJ} \mathrm{~mol}^{-1}\right]}\end{array}$ & $\begin{array}{l}{ }^{3} \Delta G^{0} \\
{\left[\mathrm{~kJ} \mathrm{~mol}^{-1}\right]}\end{array}$ & $\begin{array}{l}{ }^{1} \Delta G^{0} \\
{\left[\mathrm{~kJ} \mathrm{~mol}^{-1}\right]}\end{array}$ & $\begin{array}{l}{ }^{3} \Delta G^{0} \\
{\left[\mathrm{~kJ} \mathrm{~mol}{ }^{-1}\right]}\end{array}$ \\
\hline 1C & 2.14 & -20 & 6 & -20 & 5 \\
\hline $1 \mathrm{~T}$ & 2.11 & -22 & 3 & -23 & 2 \\
\hline $\mathbf{1 A}$ & 1.96 & -37 & -12 & -38 & -12 \\
\hline 1G & 1.49 & -82 & -57 & -83 & -58 \\
\hline
\end{tabular}
nucleobases by the $S_{1}\left({ }^{1} \Delta G^{0}\right)$ and $T_{1}$ state $\left({ }^{3} \Delta G^{0}\right)$ of $\mathrm{BP}$ in $\mathrm{MeOH}$ and in $\mathrm{H}_{2} \mathrm{O}$
The Marcus equation is highly nonlinear and an intuitive prediction of the dependence of ${ }^{1} k_{\mathrm{ET}}$ on $\Delta G^{0}$ is difficult. From a detailed consideration we conclude that only $\lambda$ values around $62 \mathrm{~kJ} \mathrm{~mol}^{-1}$ and $H_{\mathrm{AB}}=1.49 \mathrm{meV}\left(12 \mathrm{~cm}^{-1}\right)$ allow a good reproduction of the experimental observations. We find that the ET process already seems to be in the Marcus inverted regime for 1G, in agreement with the reported behaviour in DNA hairpin structures. ${ }^{52}$

Another approach to verify an ET process between the nucleobase and BP in its $\mathrm{S}_{1}$ state is the spectroscopic determination and analysis of the triplet yield $\Phi_{\mathrm{T}}$ for each compound. If a deactivation pathway of the BP $S_{1}$ state competes with ISC, the triplet quantum yield $\Phi_{\mathrm{T}}$ of BP should be reduced. For the measurements in $\mathrm{MeOH}$ we are indeed able to quantify $\Phi_{\mathrm{T}}$. By dividing the integrated transient absorption of an $\mathrm{S}_{1}$ specific absorption band (303-350 nm, blue shaded area in Fig. 6) directly after excitation $(t=0.5 \mathrm{ps})$ by the integrated transient absorption of a $\mathrm{T}_{1}$ specific absorption band (450-600 nm, red shaded area) at a pump-probe delay of $\Delta t=100 \mathrm{ps}$ we calculate $\Phi_{\mathrm{T}}$ for each compound. The yield $\Phi_{\mathrm{T}}$ determined in this way (see Table 2 ) is in good agreement with the triplet yield calculated from the values of ${ }^{1} k_{\mathrm{ET}}$ and $k_{\mathrm{ISC}}$ determined above according to eqn (3)

$$
\Phi_{\mathrm{T}}=\frac{k_{\mathrm{ISC}}}{{ }^{1} k_{\mathrm{ET}}+k_{\mathrm{ISC}}}
$$

Turning to the triplet manifold, we note that in $\mathrm{MeOH}$ there are seemingly no charge transfer processes between the BP moiety and the attached nucleobase which are faster than a nanosecond. This stands in contrast to the results in water (see below) and

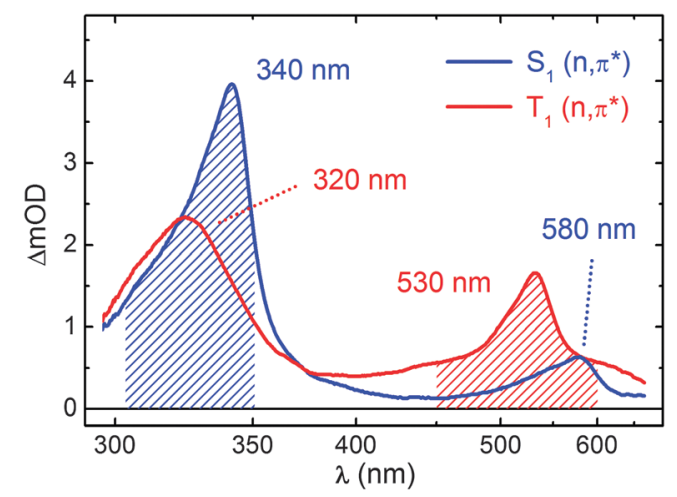

Fig. 6 SAS of $\mathrm{BP}_{1}$ (blue) and $\mathrm{T}_{1}$ state (red) after $358 \mathrm{~nm}$ excitation of $1 \mathrm{C}$ in $\mathrm{MeOH}$. The shaded integrals were used to determine the relative triplet yield. 
implies gating of electron transfer by conformational changes, as discussed later in the theoretical part. The calculated $\Delta G^{0}$ values for $\mathbf{1 A}$, and particularly $\mathbf{1 C}$ and $\mathbf{1 T}$ for the $\mathrm{BP} \mathrm{T}_{1}$ state are close to zero or even positive in $\mathrm{MeOH}$ and effectively prohibit an ET process. This is in agreement with our measurements. For 1G we see a weak signature of the BP anion around $333 \mathrm{~nm}$ toward the end of the probe range that might well be due to slow and inefficient ET between guanine and the BP triplet state.

The species associated spectra (SAS) of compound 1C in $\mathrm{MeOH}$ (Fig. 6) are determined from the decay associated difference spectra (DADS) directly obtained from a global fit. The species decaying with $9 \mathrm{ps}$ is assigned as the $\mathrm{S}_{1}$ state, the species with the long lasting spectral contribution as the $\mathrm{T}_{1}$ state. The corresponding spectra of all other compounds are identical within experimental precision to these two spectra. The SAS corroborate our qualitative discussion given above that the early spectral features are due to the initially populated $S_{1}$ state, and the later ones to the $\mathrm{T}_{1}$ state. The lack of sizable anion signatures in the transient spectra indicates that the back ET is considerably faster than the forward rates of $3 \times 10^{9}$ to $2.5 \times 10^{10} \mathrm{~s}^{-1}$.

It was shown above that the phosphorescence spectrum of the BP chromophore in $\mathbf{1}$ is affected by water as a solvent (Fig. 4). This influence is, however, not observed for the nucleosides $\mathbf{1 X}$. To clarify the kinetics behind this observation, a second series of measurements was performed in $\mathrm{H}_{2} \mathrm{O}$, the natural environment for DNA. Directly after excitation of compounds $\mathbf{1 X}$ we observe the transient signatures of the $\mathrm{BP}_{1}$ state as already observed in $\mathrm{MeOH}$. The lifetime of the $\mathrm{S}_{1}$ state reduces with decreasing standard oxidation potential $E^{0}$ of the adjacent nucleobase, similar to what is observed in $\mathrm{MeOH}$. In contrast to the measurements in $\mathrm{MeOH}$, we also find changes in the transient spectra for $\mathbf{1 G}$ and $\mathbf{1 A}$ at delay times in the $100 \mathrm{ps}$ regime (Fig. 7). They have to be interpreted as a significantly reduced lifetime of the $\mathrm{T}_{1}$ state due to ET from the adjacent nucleobase. The spin multiplicity of the dinucleotide is conserved in this process and the formed intramolecular radical ion pair still exhibits triplet character. ${ }^{53}$ All determined time constants are summarized in Table 2.

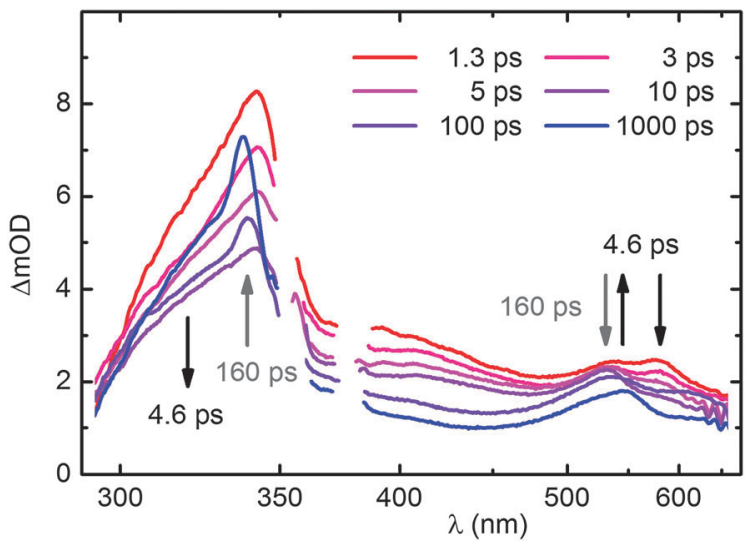

Fig. 7 TA spectra of compound $\mathbf{1 G}$ in $\mathrm{H}_{2} \mathrm{O}$ for selected pump-probe delays after $358 \mathrm{~nm}$ excitation. The wavelength region containing scattered light of the pump pulse was clipped.
The dominant influence of the nucleobases in compounds $1 \mathrm{X}$ dissolved in $\mathrm{H}_{2} \mathrm{O}$ is the singlet ET. A plot of ${ }^{1} k_{\mathrm{ET}} v s .{ }^{1} \Delta G^{0}$ (see Fig. SI-17, ESI $\dagger$ ) confirms the picture which we already obtained from the measurements in $\mathrm{MeOH}$. We also applied the Marcus equation for evaluation of the dependence of ${ }^{1} k_{\mathrm{ET}}$ on ${ }^{1} \Delta G^{0}$ in $\mathrm{H}_{2} \mathrm{O}$. The reorganization energy of $\lambda=67 \mathrm{~kJ} \mathrm{~mol}^{-1}$ $(0.70 \mathrm{eV})$ is close to the value found for $\mathrm{MeOH}$, while the fitted electronic coupling strength is $H_{\mathrm{AB}}=2.64 \mathrm{meV}\left(21 \mathrm{~cm}^{-1}\right)$. Again, the parameter $H_{\mathrm{AB}}$ is just an effective value and will be later discussed in connection with the distribution of donor acceptor distances.

Due to the decay dynamics of the triplet state, i.e. the significant ET in the triple state, we were not able to quantify the triplet yield in the same fashion as for $\mathrm{MeOH}$. The transient spectra of samples 1G and 1A in $\mathrm{H}_{2} \mathrm{O}$ show a decay of the triplet spectrum with simultaneously upcoming sharp spectral signatures of the $\mathrm{BP}$ radical anion at $335 \mathrm{~nm}$ (Fig. 7 and 8). The assignment of the spectral features to the spectrum of the BP radical anion is done in accord with the literature. ${ }^{46}$ The spectra in Fig. 7 for compound 1G are exemplary also for compound $\mathbf{1 A}$ with the exception of the precise values of the time constants.

To further clarify the charge transfer dynamics between the nucleobase and the BP $\mathrm{T}_{1}$ state it seems reasonable to analyze the dynamics of the integrated transient absorption between 300 and $340 \mathrm{~nm}$ due to well distinguishable absorption characteristics of the excited singlet, triplet, and radical anion state in this spectral region. A multi-exponential fit provides within experimental error the same time constants as already obtained from the global data analysis. The data points and the corresponding multi-exponential fit curves are depicted in Fig. 8. All data have a sizable offset, i.e. the integral signal does not decay to zero within the observation window. This is due to the lasting charge separation in the triplet state. Such a long living CT state is not unexpected, due to the remaining spin correlation in the intramolecular radical ion pair. ${ }^{53}$

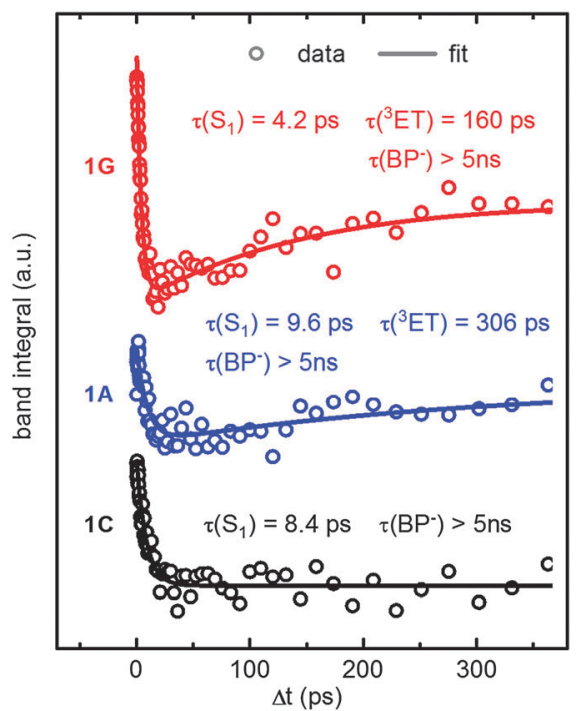

Fig. 8 Integrated transient absorption from 300 to $340 \mathrm{~nm}$ of samples 1G, 1A and $1 \mathrm{C}$ in $\mathrm{H}_{2} \mathrm{O}$ after $358 \mathrm{~nm}$ excitation. The time constants are obtained from multi-exponential fits. 


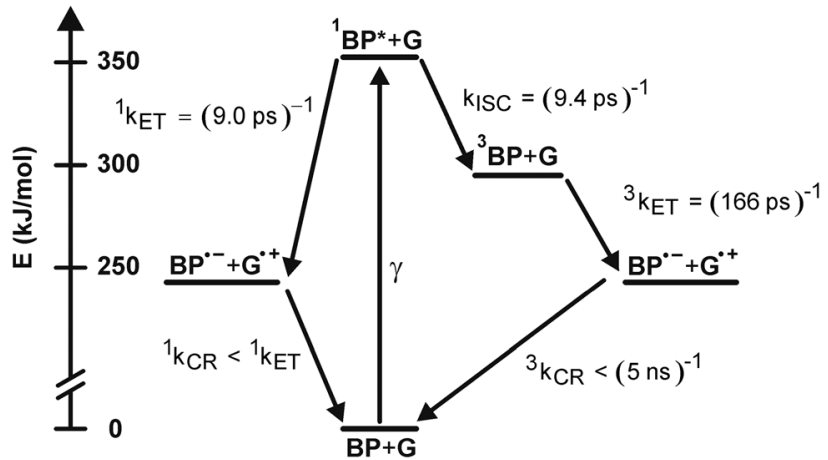

Fig. 9 Excited state energetics and kinetics of $1 \mathrm{G}$ in $\mathrm{H}_{2} \mathrm{O}$.

Despite differing sample concentrations and experimental conditions, the differing temporal behavior can be well seen from the displayed curves. Note in particular that the signal for 1C is settled after 100 ps while the signals for $\mathbf{1 G}$ and $\mathbf{1 A}$ keep increasing due to the triplet charge transfer.

All processes and the energetics of the involved levels are summarized for 1G in $\mathrm{H}_{2} \mathrm{O}$ in Fig. 9. ET is found both in the singlet and the triplet system, but the charge separation is only persistent for the triplet system. These observations are similar to the recent reports on anthraquinone-DNA conjugates. ${ }^{5 a}$ From the experimental data no solid reasoning for this striking observation can be deduced. The only argument to be invoked would be spin correlation between the radical ions and the forbidden character of the back transfer involving spin flip. It should also be remembered that ET in the triplet system is not found for $\mathrm{MeOH}$. For an explanation of these observations we turn to quantum chemical calculations.

\section{QM/MM studies for the energy landscape of $1 \mathrm{G}$}

In the following, the discussion of the results is divided into two parts discussing 1G solvated in water, and in methanol, respectively.

According to our MD simulations reported in Fig. 3, 1G in water almost exclusively exists in the folded form. We therefore have selected two snapshots ${ }^{\mathrm{a}} \mathbf{1 G}_{\mathrm{H}_{2} \mathrm{O}}$, and ${ }^{\mathrm{b}} \mathbf{1 G}_{\mathrm{H}_{2} \mathrm{O}}$ which represent the folded configuration. The calculated singlet excitation energies for these two snapshots are compiled in Table 4 (for triplet energies at the optimised ground state see Table SI-7, ESI + ).

First we concentrate on the results for the optimized ground state. The folded snapshots feature a distance between C7 and O39 of about $3.3 \AA$ As is evident from Table 4, the excitation energies of the three lowest excited states are very similar for the two individual snapshots. For all snapshots the $S_{1}, S_{2}$, and $\mathrm{S}_{3}$ states are of $\left(\mathrm{n} \pi^{*}\right),\left(\pi \pi^{*}\right)$ and $\left(\pi \pi^{*}\right)$ types. Hence, in contrast to the isolated molecule, there is no low-lying CT state in the Franck-Condon region for the folded form of $\mathbf{1 G}$ in water. Apparently, the solvent shell relaxed for the ground state destabilizes the CT state. Presumably, primarily the BP subunit
Table 4 TD-CC2 QM/MM excitation energies $\omega$ at the respective TD-DFT minima, calculated in aug-cc-pVDZ basis, respectively. $\Delta E$ corresponds to the total energy difference of the optimised $\left(n \pi^{\star}\right)$ state and the $\left(n \pi^{\star}\right)$ state at the ground state geometry $\left({ }^{\mathrm{a}} \Delta E=E_{\mathrm{tot}}\left(\mathrm{n} \pi^{\star}\right)_{\mathrm{opt}}-E_{\mathrm{tot}}\left(\mathrm{n} \pi^{\star}\right)_{\mathrm{FC}}\right)$, or of the optimised CT state and the $\left(n \pi^{\star}\right)$ state at the ground state geometry $\left({ }^{b} \Delta E=E_{\text {tot }}(C T)_{\text {opt }}-\right.$ $\left.E_{\text {tot }}\left(\mathrm{n} \pi^{\star}\right)_{\mathrm{FC}}\right)$; solvent: $\mathrm{H}_{2} \mathrm{O}$

\begin{tabular}{|c|c|c|c|c|c|c|c|c|}
\hline \multirow[b]{2}{*}{ Structure } & \multirow[b]{2}{*}{ State } & \multicolumn{2}{|c|}{ FC point } & \multicolumn{2}{|c|}{$\left(\mathrm{n} \pi^{*}\right)$ state } & \multicolumn{2}{|c|}{ CT state } & \multirow[b]{2}{*}{$\Delta E[\mathrm{eV}]$} \\
\hline & & Type & $\omega[\mathrm{eV}]$ & Type & $\omega[\mathrm{eV}]$ & Type & $\omega[\mathrm{eV}]$ & \\
\hline \multirow[t]{4}{*}{${ }^{\mathrm{a}} \mathbf{1 G}_{\mathrm{H}_{2} \mathrm{O}}$} & $\mathrm{S}_{1}$ & $\left(\mathrm{n} \pi^{*}\right)$ & 3.85 & $\left(\mathrm{n} \pi^{*}\right)$ & 3.02 & CT & 2.83 & $-0.08^{\mathrm{a}}$ \\
\hline & $\mathrm{S}_{2}$ & $\left(\pi \pi^{*}\right)$ & 4.62 & $\left(\pi \pi^{*}\right)$ & 4.57 & $\left(\mathrm{n} \pi^{*}\right)$ & 3.38 & \\
\hline & $\mathrm{S}_{3}$ & $\left(\pi \pi^{*}\right)$ & 4.75 & $\left(\pi \pi^{*}\right)$ & 4.71 & $\left(\pi \pi^{*}\right)$ & 4.18 & $-0.36^{\mathrm{b}}$ \\
\hline & $\mathrm{S}_{4}$ & - & - & CT & 4.74 & - & - & \\
\hline \multirow[t]{4}{*}{${ }^{\mathrm{b}} \mathbf{1 G}_{\mathrm{H}_{2} \mathrm{O}}$} & $\mathrm{S}_{1}$ & $\left(\mathrm{n} \pi^{*}\right)$ & 3.80 & $\left(\mathrm{n} \pi^{*}\right)$ & 3.12 & CT & 2.58 & $-0.18^{\mathrm{a}}$ \\
\hline & $\mathrm{S}_{2}$ & $\left(\pi \pi^{*}\right)$ & 4.57 & $\left(\pi \pi^{*}\right)$ & 4.51 & $\left(\mathrm{n} \pi^{*}\right)$ & 3.34 & \\
\hline & $\mathrm{S}_{3}$ & $\left(\pi \pi^{*}\right)$ & 4.61 & $\left(\pi \pi^{*}\right)$ & 4.57 & $\left(\pi \pi^{*}\right)$ & 4.14 & $-0.20^{\mathrm{b}}$ \\
\hline & $\mathrm{S}_{4}$ & - & - & CT & 4.76 & - & - & \\
\hline
\end{tabular}

is responsible for this effect (BP is not soluble in water); the solvent shell around BP changes considerably when optimizing the geometry on the potential energy surface of the CT state.

The density difference plots and dipole differences (relative to the ground state) corresponding to ${ }^{\mathrm{a}} \mathbf{G}_{\mathrm{H}_{2} \mathrm{O}}$ are displayed in Fig. 10 (for the relevant molecular orbital contributions $c f$. Fig. SI-29-a, ESI $\dagger$ ). The density difference of the $S_{1}$ state looks similar to that of the $S_{1}$ state of the isolated system (Fig. 2).

It has the character of a $\left(\mathrm{n} \pi^{*}\right)$ transition with a dipole moment difference of about 1.9 D. The $S_{1} \leftarrow S_{0}$ excitation energies of the two snap shots amount to $320-330 \mathrm{~nm}$, which fits the experimental value of $329 \mathrm{~nm}$ quite well. Relative to the gas phase values, the solvated molecules appear to be blue shifted by $10-20 \mathrm{~nm}$.

a)

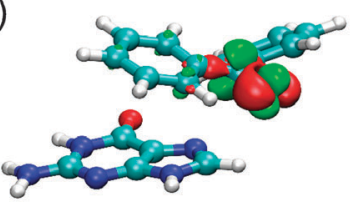

b)

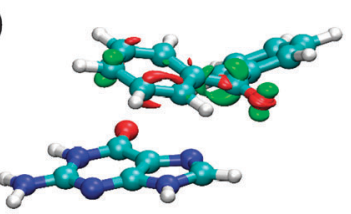

c)

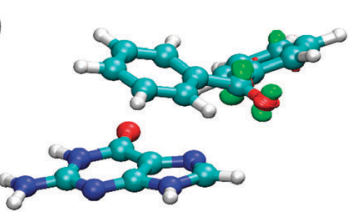

Fig. 10 Electron density difference plots for the first three excited singlet states of ${ }^{\mathrm{a}} \mathbf{1 G}_{\mathrm{H}_{2} \mathrm{O}}$, calculated with TD-CC2 in the aug-cc-pVDZ basis in the QM/MM framework at the Franck-Condon point. Isosurfaces are plotted for \pm 0.005 a.u., red refers to a decrease, green to an increase in the density upon excitation. (a) $\mathrm{S}_{1}:\left(\mathrm{n} \pi^{\star}\right)$ state, $|\Delta \mu|=1.86 \mathrm{D}$ (b) $\mathrm{S}_{2}:\left(\pi \pi^{\star}\right)$ state, $|\Delta \mu|=2.43 \mathrm{D}$ (c) $\mathrm{S}_{3}:\left(\pi \pi^{\star}\right)$ state, $|\Delta \mu|=2.46 \mathrm{D}$. 
$S_{2}$ and $S_{3}$ are quite well separated from $S_{1}$, but the gap between $S_{2}$ and $S_{3}$ is very small. These are $\left(\pi \pi^{*}\right)$ states with dipole differences of 2.4-2.5 D.

Geometry optimization on the $S_{1}$ state surface, which was done in order to check if on the route downhill to the $S_{1}$ state minimum a crossing with a CT state occurs, showed that the above mentioned distances $d(\mathrm{C} 7-\mathrm{O} 39)$ compared to the ground state optimized ones (see Table SI-5, ESI $\dagger$ ), do not alter much. Relative to the FC point the total energy drops slightly by $0.1 \mathrm{eV}\left({ }^{\mathrm{a}} \mathbf{1}_{\mathrm{G}_{2} \mathrm{O}}\right)$ and $0.2 \mathrm{eV}\left({ }^{\mathrm{b}} \mathbf{1 G}_{\mathrm{H}_{2} \mathrm{O}}\right)$. Similarly as at the Franck-Condon point the $\mathrm{S}_{1}, \mathrm{~S}_{2}$, and $\mathrm{S}_{3}$ states are again of $\left(n \pi^{*}\right),\left(\pi \pi^{*}\right)$, and $\left(\pi \pi^{*}\right)$ type, respectively. Again, $S_{2}$ and $S_{3}$ are energetically rather close, while the gap between $S_{1}$ on the one hand, and $S_{2}, S_{3}$ on the other hand, is fairly large. However, a CT state now comes into play as the $\mathrm{S}_{4}$ state.

Therefore, for ${ }^{\mathrm{a}} \mathbf{1 G}_{\mathrm{H}_{2} \mathrm{O}}$ and ${ }^{\mathrm{b}} \mathbf{1 G}_{\mathrm{H}_{2} \mathrm{O}}$ further geometry optimizations on this CT state surface were performed. The relevant density difference plots and dipole moment differences related to ${ }^{\mathrm{a}} \mathbf{1 G}_{\mathrm{H}_{2} \mathrm{O}}$ are given in Fig. 11 . The CT state minimum for the ${ }^{\mathrm{a}} \mathbf{1 G}_{\mathrm{H}_{2} \mathrm{O}}$ and ${ }^{\mathrm{b}} \mathbf{1 G}_{\mathrm{H}_{2} \mathrm{O}}$ snapshots is $\Delta E=0.4$ and $\Delta E=0.2 \mathrm{eV}$ below the FC point ( $c f$. Table 4 ), hence at about the same energy as the $\mathrm{S}_{1}$ state minimum $\left({ }^{\mathrm{b}} \mathbf{1}_{\mathrm{H}_{2} \mathrm{O}}\right)$, or slightly below $\left({ }^{\mathrm{a}} \mathbf{1}_{\mathrm{H}_{2} \mathrm{O}}\right)$. This allows population transfer from the excited $S_{1}$ state to the dark CT state with reasonable efficiency.

Interestingly, the $S_{3}\left(\pi \pi^{*}\right)$ state at the CT state minimum now corresponds to a local excitation on the $\mathrm{G}$ subunit with a relatively large dipole moment difference (relative to the ground state at that geometry) of more than $10 \mathrm{D}$.

In the course of the optimization on the CT state surface, the distance between the oxygen $\mathrm{O} 39$ of the carbonyl group of BP
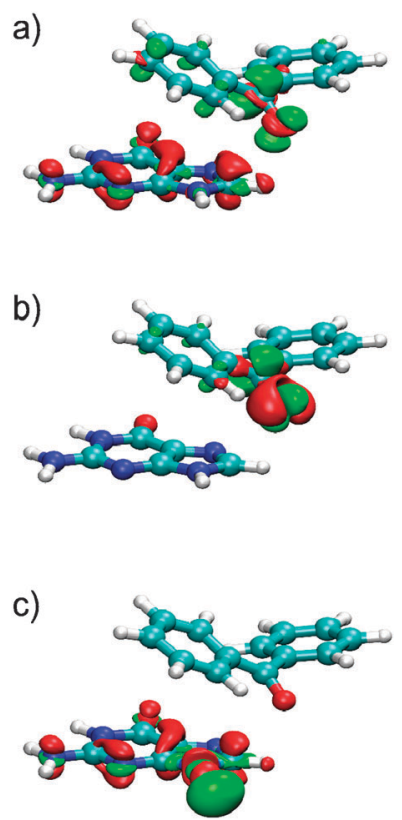

Fig. 11 Electron density difference plots for the first three excited singlet states of ${ }^{\mathrm{a}} \mathbf{1 G}_{\mathrm{H}_{2} \mathrm{O}}$, calculated with TD-CC2 in the aug-cc-pVDZ basis in the QM/MM framework, at the TD-DFT/BHLYP CT minimum. Isosurfaces are plotted for \pm 0.005 a.u., red refers to a decrease, green to an increase in the density upon excitation. (a) $\mathrm{S}_{1}$ : CT state; $|\Delta \mu|=15.59 \mathrm{D}$ (b) $\mathrm{S}_{2}:\left(\mathrm{n} \pi^{\star}\right)$ state; $|\Delta \mu|=2.34 \mathrm{D}$ (c) $\mathrm{S}_{3}$ : $\left(\pi \pi^{\star}\right)$ type state; $|\Delta \mu|=14.24 \mathrm{D}$. and the $\mathrm{C} 7$ atom of the $\mathrm{G}$ decreases substantially from $3.22 \AA$ $\left({ }^{\mathrm{a}} \mathbf{1 G}_{\mathrm{H}_{2} \mathrm{O}}\right)$ or $3.39 \AA\left({ }^{\mathrm{b}} \mathbf{1 G}_{\mathrm{H}_{2} \mathrm{O}}\right)$ for the ground state minimum to $2.73 \AA\left({ }^{\mathrm{a}} \mathbf{1 G}_{\mathrm{H}_{2} \mathrm{O}}\right)$ or $2.93 \AA\left({ }^{\mathrm{b}} \mathbf{1 G}_{\mathrm{H}_{2} \mathrm{O}}\right)$ for the optimized CT state. The shortening of this distance might indicate a possible subsequent proton transfer, equalizing the charge separation induced by the preceding electron transfer ( $c f$. Fig. 11).

From the MD trajectory in methanol three individual snapshots were taken, i.e. two snapshots ${ }^{\mathrm{a}} \mathbf{1 G}_{\mathrm{MeOH}}$ and ${ }^{\mathrm{b}} \mathbf{1 G}_{\mathrm{MeOH}}$, which represent the characteristic unfolded conformation, and a representative snapshot ${ }^{\mathrm{c}} \mathbf{1 G}_{\mathrm{MeOH}}$ for a folded conformation in methanol. As already discussed, 1G exists in the methanol environment predominantly in the unfolded conformation. The folded conformation was selected to check if in such a geometry a transition to the charge transfer state is also possible in the methanol environment. For the optimized ground state geometries, the distance $d(\mathrm{C} 7-\mathrm{O} 39)$ of the unfolded conformers ranges between 14 and $17 \AA$, while that of the folded conformer amounts to about $3.1 \AA$ (see Fig. SI-28, ESI $\dagger$ ). This is comparable to the folded snapshots in water. The excitation energies of the three lowest excited singlet states obtained by the QM/MM calculations are compiled in Table 5 (for triplet energies at the optimised ground state see Table SI-7, ESI $\dagger$ ).

Evidently, the excitation energies of the two unfolded snapshots are very similar: the first excitation corresponds to a $\left(n \pi^{*}\right)$ state. As is the case also in water, the calculated first excitation energies fit the experimental value of $329 \mathrm{~nm}$ quite well. Again, similar to the spectrum in water, the $\left(n \pi^{*}\right)$ state is well separated from the $S_{2}$ and $S_{3}$ states, which in turn are rather close together. $S_{2}$ and $S_{3}$ are $\left(\pi \pi^{*}\right)$ states. In contrast to $1 \mathbf{G}$ solvated in water the $\mathrm{S}_{3}$ state now corresponds to a local excitation on the G, rather than on the BP subunit.

The $S_{1}$ state of the folded snapshot also has $\left(n \pi^{*}\right)$ character with similar excitation energy as the $S_{1}$ states of the two unfolded snapshots, and similar also to the $S_{1}$ state excitation energies of the two folded snapshots in water (vide supra). It thus appears that the $S_{1}$ state is hardly affected by the different solvent environments and the dynamics of folding and unfolding. The $S_{2}$ state of the folded snapshot on the other hand has CT character: in contrast to the case of the water environment and

Table 5 TD-CC2 QM/MM excitation energies $\omega$ at the respective TD-DFT minima, calculated in aug-cc-pVDZ basis, respectively. $\Delta E\left(\Delta E=E_{\text {tot }}(C T)_{\text {opt }}-\right.$ $\left.E_{\text {tot }}\left(n \pi^{\star}\right)_{F C}\right)$ corresponds to the total energy difference of the optimised CT state and the $\left(n \pi^{\star}\right)$ at the FC point; solvent: $\mathrm{MeOH}$

\begin{tabular}{|c|c|c|c|c|c|c|c|c|}
\hline \multirow[b]{2}{*}{ Structure } & \multirow[b]{2}{*}{ State } & \multicolumn{2}{|c|}{ FC point } & \multicolumn{2}{|c|}{$\left(\mathrm{n} \pi^{*}\right)$ state } & \multicolumn{2}{|c|}{ CT state } & \multirow[b]{2}{*}{$\Delta E[\mathrm{eV}]$} \\
\hline & & Type & $\omega[\mathrm{eV}]$ & Type & $\omega[\mathrm{eV}]$ & Type & $\omega[\mathrm{eV}]$ & \\
\hline${ }^{\mathrm{a}} \mathbf{1 G}_{\mathrm{MeOH}}$ & $\begin{array}{l}S_{1} \\
S_{2} \\
S_{3}\end{array}$ & $\begin{array}{l}\left(\mathrm{n} \pi^{*}\right) \\
\left(\pi \pi^{*}\right) \\
\left(\pi \pi^{*}\right)\end{array}$ & $\begin{array}{l}3.78 \\
4.70 \\
4.82\end{array}$ & $\begin{array}{l}\left(\mathrm{n} \pi^{*}\right) \\
\left(\pi \pi^{*}\right) \\
\left(\pi \pi^{*}\right)\end{array}$ & $\begin{array}{l}3.15 \\
4.66 \\
4.71\end{array}$ & - & & - \\
\hline${ }^{\mathrm{b}} \mathbf{1 G}_{\mathrm{MeOH}}$ & $\begin{array}{l}S_{1} \\
S_{2} \\
S_{3}\end{array}$ & $\begin{array}{l}\left(\mathrm{n} \pi^{*}\right) \\
\left(\pi \pi^{*}\right) \\
\left(\pi \pi^{*}\right)\end{array}$ & $\begin{array}{l}3.84 \\
4.58 \\
4.65\end{array}$ & $\begin{array}{l}\left(\mathrm{n} \pi^{*}\right) \\
\left(\pi \pi^{*}\right) \\
\left(\pi \pi^{*}\right)\end{array}$ & $\begin{array}{l}3.13 \\
4.43 \\
4.61\end{array}$ & - & & - \\
\hline${ }^{\mathrm{c}} \mathbf{1 G}_{\mathrm{MeOH}}$ & $\begin{array}{l}S_{1} \\
S_{2} \\
S_{3}\end{array}$ & $\begin{array}{l}\left(\mathrm{n} \pi^{*}\right) \\
\mathrm{CT} \\
\left(\pi \pi^{*}\right)\end{array}$ & $\begin{array}{l}3.96 \\
4.28 \\
4.61\end{array}$ & - & & $\begin{array}{l}\text { CT } \\
\left(\mathrm{n} \pi^{*}\right) \\
\left(\pi \pi^{*}\right)\end{array}$ & $\begin{array}{l}2.28 \\
3.48 \\
3.75\end{array}$ & -0.84 \\
\hline
\end{tabular}


a)

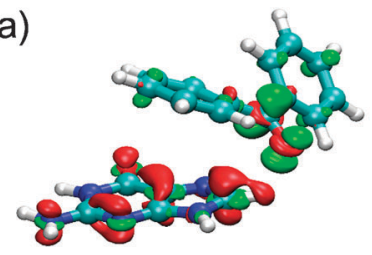

b)

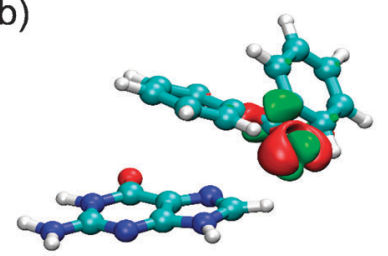

c)

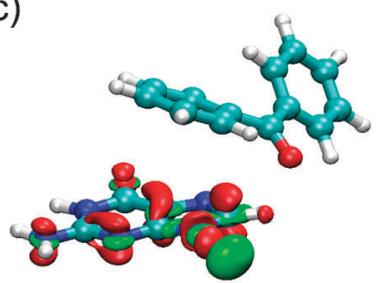

Fig. 12 Electron density difference plots for the first three excited singlet states of ${ }^{\mathrm{C}} \mathbf{1} \mathbf{G}_{\mathrm{MeOH}}$, calculated with TD-CC2 in the aug-cc-pVDZ basis in the QM/MM framework, at the TD-DFT/BHLYP CT minimum. Isosurfaces are plotted for \pm 0.005 a.u., red refers to a decrease, green to an increase in the density upon excitation. (a) $\mathrm{S}_{1}:$ CT state; $|\Delta \mu|=19.75 \mathrm{D}$ (b) $\mathrm{S}_{2}:\left(\mathrm{n} \pi^{\star}\right)$ state; $|\Delta \mu|=2.04 \mathrm{D}$ (c) $\mathrm{S}_{3}$ : $\left(\pi \pi^{\star}\right)$ type state; $|\Delta \mu|=11.11 \mathrm{D}$

similar to the case of the isolated system, we find a low-lying CT state already at the FC point, only $0.32 \mathrm{eV}$ above the $\mathrm{S}_{1}$ state. Here, in contrast to the water environment, the solvent shell relaxed for the electronic ground state also stabilizes the CT state (note that BP is soluble in $\mathrm{MeOH}$, but not in water). The density difference plots, dipole differences and relevant molecular orbitals related to ${ }^{\mathrm{a}} \mathbf{1} \mathbf{G}_{\mathrm{MeOH}}$ and ${ }^{\mathrm{c}} \mathbf{1 G}_{\mathrm{MeOH}}$ are given in the ESI $\dagger$ (Fig. SI-29-b, SI-29-c and SI-30).

In analogy to the calculations performed for $\mathbf{1 G}$ in water geometry optimizations on the $\mathrm{S}_{1}$ state surface were also performed. For the two unfolded conformers the resulting geometries overall look quite similar to those of the ground state. The distance $d(\mathrm{C} 7-\mathrm{O} 39)$ measuring the degree of unfolding does not alter much.

The $S_{1}$ state excitation energy decreases by about $0.7 \mathrm{eV}$ on going from the ground state to the $S_{1}$ state minimum, which is very similar to the situation of $\mathbf{1 G}$ in water. The characters of the three lowest excited states at the $S_{1}$ state minimum geometry are $\left(\mathrm{n} \pi^{*}\right),\left(\pi \pi^{*}\right)$, and $\left(\pi \pi^{*}\right)$, respectively, with the $\mathrm{S}_{3}$ state corresponding to a local excitation on the BP, rather than on the $\mathrm{G}$ subunit. No CT state occurs.

The situation is entirely different for the folded snapshot: during optimization on the $S_{1}$ state surface, the $S_{1}$ state switches character from $\left(\mathrm{n} \pi^{*}\right)$ to CT. An optimization of the respective state with $\left(\mathrm{n} \pi^{*}\right)$ character was not possible. The CT state minimum energy geometry definitely constitutes the deepest minimum; the total energy drops by about $0.8 \mathrm{eV}$ on going from the FC point to the CT state minimum ( $c f$. Table 5). Furthermore, the $d(\mathrm{C} 7-\mathrm{O} 39)$ distance contracts from 3.1 to $2.85 \AA$.

Population transfer from the excited (n $\left.\pi^{*}\right)$ to the CT state thus appears to be rather efficient.

The $S_{2}$ state at the CT state minimum geometry has now $\left(n \pi^{*}\right)$ character. It is similar to the $\mathrm{S}_{1}$ state at the Franck-Condon point with a dipole moment difference of $|\Delta \mu|=2.04 \mathrm{D}$. The $\mathrm{S}_{3}$ state now corresponds to a $\left(\pi \pi^{*}\right)$ state localized on the G subunit with a very high dipole moment difference of $|\Delta \mu|=11.11 \mathrm{D}$, which looks quite similar to the $S_{3}$ state at the CT minimum of $\mathbf{1 G}$ in water ( $c f$. Fig. 10c). The density difference plots and dipole moment differences related to the $\mathrm{S}_{1}$ (CT) state minimum of ${ }^{\mathrm{c}} \mathbf{1 G}_{\mathrm{MeOH}}$ are given in Fig. 12.

From the QM/MM results discussed in this section the following conclusions, independent of the solvent environment, can be drawn: (i) for 1G in the unfolded form no low-lying CT state is found, which could quench the excited ( $\left.\mathrm{n} \pi^{*}\right)$ singlet state. Here, the lifetime of the excited $\left(n \pi^{*}\right)$ state is primarily determined by intersystem crossing to the triplet state. (ii) For 1G in the folded form, on the other hand, there indeed exists a low-lying CT state in some regions of the configuration space, which could quench the excited $\left(n \pi^{*}\right)$ singlet state. In the methanol environment, provided that $\mathbf{1 G}$ adopts the folded form, the transition from the (n $\left.\pi^{*}\right)$ to the CT state should be more efficient than in the water environment, where the conical intersection apparently does not cross the downhill path from the $\mathrm{FC}$ to the $\mathrm{S}_{1}$ state minimum point. However, in the methanol environment 1G predominantly exists in the unfolded form, hence transition from the $\left(\mathrm{n} \pi^{*}\right)$ to the CT state is overall not very frequent. On the other hand, in water, 1G predominantly exists in the folded form, but the state transition itself is less efficient. We conclude that in both environments quenching of the $S_{1}$ state due to population transfer to the dark CT state can occur, but with rather low efficiency due to different reasons. Nevertheless, the calculations predict that the lifetime of the $\left(\mathrm{n} \pi^{*}\right)$ singlet state is not solely governed by ISC, as is also seen in the experiments.

\section{Discussion}

The major goal of this study is to elucidate the conformational influence on photoinduced charge transfer in four different dinucleotides that were designed and synthesized as models to reduce the complexity of the conformational manifold present in double-stranded DNA. The first advantage of the models is that the influence of each of the four different DNA bases on the photophysical behavior of BP can be studied exclusively. Therefore this approach provides the best chance to get a profound understanding of BP interactions in nucleic acids. The second advantage is that two different solvents can be chosen since the dinucleotides are soluble in $\mathrm{H}_{2} \mathrm{O}$ and $\mathrm{MeOH} . \mathrm{H}_{2} \mathrm{O}$ represents the typical solvent for oligonucleotides in a pH-controlled buffer, whereas $\mathrm{MeOH}$ mimics a protic solvent ${ }^{54}$ approximately the polarity of the inner part of double stranded DNA (DNA base stack). BP as an artificial nucleoside, allows selective excitation of its photochemically important ${ }^{1}\left(\mathrm{n} \pi^{*}\right)$ transition outside the typical nucleic acid absorption range $(>300 \mathrm{~nm})$, which is an important prerequisite to use the chromophore in photochemistry and photobiology. 
By employing ultrafast transient absorption spectroscopy it was found that the lifetime of the excited $\left(n \pi^{*}\right)$ singlet state is primarily determined by fast intersystem crossing (ISC) to the lowest triplet state. In $\mathrm{MeOH}$ the reference compound 1 features a somewhat reduced $S_{1}\left(n \pi^{*}\right)$ singlet lifetime of $9.4 \mathrm{ps}$ relative to the $\mathrm{BP}$ monomer $(12.5 \mathrm{ps})$. Relative to the reference $\mathbf{1}$, the $S_{1}$ lifetimes of the dinucleotides $\mathbf{1 X}$ are further decreased. This effect is most pronounced for $1 \mathrm{G}(7.6 \pm 0.5 \mathrm{ps})$. These observations indicate a singlet electron transfer in $\mathbf{1 X}$, and indeed ET from a DNA nucleobase to the $\mathrm{S}_{1}$ state of BP in $\mathrm{MeOH}$ occurs at a rate between $3 \times 10^{9}$ and $25 \times 10^{9} \mathrm{~s}^{-1}$, which competes with the ISC process. In $\mathrm{H}_{2} \mathrm{O}$ the lifetime of the $\mathrm{S}_{1}$ state reduces with decreasing standard oxidation potential $E^{0}$ of the adjacent nucleobase similar to what is observed in $\mathrm{MeOH}$. Changing the solvent from $\mathrm{MeOH}$ to $\mathrm{H}_{2} \mathrm{O}$ has the most dramatic effect in 1G: the $\left(\mathrm{n} \pi^{*}\right)$ state lifetime of $\mathbf{1 G}$ now drops to $4.6 \mathrm{ps}$, while the lifetimes of the other dinucleotides $\mathbf{1 X}$ with $\mathbf{X}=\mathbf{T}, \mathbf{A}$ or $\mathbf{C}$ are much less affected. This can again be attributed to a transition from the $S_{1}\left(n \pi^{*}\right)$ to a singlet charge transfer (CT) state. The interpretation of the $S_{1}$ lifetime shortening is strongly supported by the calculations that find indeed an energetically low lying CT state for $\mathbf{1 G}$ in both solvents. For $\mathbf{1 A}$ and $\mathbf{1 G}$ in $\mathrm{H}_{2} \mathrm{O}$ we even find clear spectroscopic signatures for the BP anion moiety. As the triplet energy is well below the singlet one, an ET from the $\mathrm{S}_{1}$ state must have a large driving force.

The population transfer to the CT state is competitive with the ISC for the $1 \mathrm{G}$ dinucleotide in $\mathrm{H}_{2} \mathrm{O}$, with a $51 \%$ yield. The reorganization energy was determined to be $\lambda=0.62 \mathrm{eV}$ in $\mathrm{MeOH}$ and is nearly identical $(\lambda=0.67 \mathrm{eV})$ in the more polar $\mathrm{H}_{2} \mathrm{O}$. Considering the conformational distribution, we find a value for the electronic coupling of $H_{\mathrm{AB}}=3 \mathrm{meV}$. In comparison to previously reported values for electronic coupling ${ }^{52}$ the presented value appears to be rather small. It became clear that the solvent controls the conformational distribution and thereby gates the charge transfer due to differences in distance and stacking. Therefore our results represent a full account of the photophysical properties of the singlet and triplet states of the BP chromophore in the context of each of the four different DNA bases.

The theoretical work focuses on the dynamics and electronic structure of the dinucleotide $\mathbf{1 G}$, which is the most interesting dinucleotide. Extensive molecular mechanics (MM) and hybrid quantum mechanics/molecular mechanics (QM/MM) calculations were carried out for $\mathbf{1 G}$ solvated in $\mathrm{MeOH}$ and water. It turns out that $\mathbf{1 G}$ solvated in water predominantly exists in a folded form with the $\mathrm{G}$ ring system undergoing $\pi$-stacking with one of the rings of BP. The distance between the two ring systems amounts to 5-6 Å. Furthermore, there indeed exists a low-lying CT state, corresponding to the lowest-lying excited singlet state in some part of configuration space. This CT state shifts electron density from the $\mathrm{G}$ to the BP subsystem. No conical intersection seam between CT and $S_{1}\left(n \pi^{*}\right)$ state is found in the vicinity of the Franck-Condon point, or on the path downhill on the potential energy surface towards the $S_{1}$ state minimum. This speaks for a possible additional singlet to singlet decay channel for the $S_{1}$ $\left(\mathrm{n} \pi^{*}\right)$ state, which however is not so efficient to outperform ISC, as is indeed observed experimentally. For $\mathbf{1 G}$ solvated in $\mathrm{MeOH}$, on the other hand, the unfolded form, with the $\mathrm{G}$ and the $\mathrm{BP}$ subunits being separated by about $15 \AA$, is the favored arrangement, for which no such decay channel to a CT state exists. However, there are some rather rare occurrences of also a folded form of $\mathbf{1 G}$ in methanol in the MD trajectory. For these a CT state can indeed be observed. The decay channel for the $\left(\mathrm{n} \pi^{*}\right)$ state via this CT state appears to be much more efficient than in the water environment, since the conical intersection is encountered directly on the downhill path from the FranckCondon point on the $\left(n \pi^{*}\right)$ state surface. Nevertheless, since the $1 G$ predominantly exists in an unfolded form, population transfer from the ( $\mathrm{n} \pi^{*}$ ) to the CT state surface remains inefficient in the methanol environment.

By combining these theoretical results with the experimental work now a complete picture emerges. The almost twice as large lifetime $\tau_{\mathrm{S}_{1}}$ of $\mathbf{1 G}$ in $\mathrm{MeOH} v s$. water ( $c f$. Table 2) can thus be explained by the different distances between the donor and the acceptor, which are controlled by the solvent: CT in closed geometry of $\mathbf{1 G}\left(\mathrm{H}_{2} \mathrm{O}\right)$ on a time scale of 9 ps against CT in the open geometry of $\mathbf{1 G}(\mathrm{MeOH})$ on a $40 \mathrm{ps}$ time scale. Of course, some smaller fraction of the solvated molecules in $\mathrm{MeOH}$ assumes the folded form where the channel to the CT state is open. This explains the somewhat reduced $S_{1}\left(n \pi^{*}\right)$ state lifetime of $7.6 \pm 0.5 \mathrm{ps}$ of $\mathbf{1 G}$ in $\mathrm{MeOH}$ compared to that of $\mathbf{1}$.

Finally, the photophysical behavior of the triplet state of the BP moiety in $\mathbf{1 X}$ is explored, since the triplet state is predominantly applied in photochemical and photobiological reactions. As already discussed in the previous section, singlet CT processes between the DNA base and the BP chromophore of dinucleotides $\mathbf{1 X}$ solvated in $\mathrm{MeOH}$ occur on a time scale of tens of ps, which is comparable to the rate of ISC. In contrast, the lifetimes of the BP $\mathrm{T}_{1}$ state of the dinucleotides $\mathbf{1 X}$ in $\mathrm{MeOH}$ remain unaffected on the ps time-scale by the adjacent nucleobase. There seems to be no charge transfer taking place between the $\mathrm{BP} \mathrm{T}_{1}$ state and the attached nucleobase which is faster than a couple of nanoseconds. And again, the situation in $\mathrm{H}_{2} \mathrm{O}$ looks significantly different from $\mathrm{MeOH}$. The lifetimes of the $\mathrm{T}_{1}$ state of $\mathbf{1 C}$ and $\mathbf{1 T}$ remain unaffected while the $\mathrm{T}_{1}$ lifetimes of $\mathbf{1 G}$ and $\mathbf{1 A}$ are significantly reduced due to CT from the adjacent nucleobase. In fact, the transient absorption of $\mathbf{1 G}$ and 1A shows the decay of the triplet spectrum concomitantly with the upcoming spectral signature of the BP radical anion. Charge transfer from the $\mathrm{T}_{1}$ state occurs in $\mathrm{H}_{2} \mathrm{O}$ with $3.2 \times 10^{9}$ to $6.0 \times 10^{9} \mathrm{~s}^{-1}$. In contrast to the fast recombination in the singlet manifold the CT state, which is populated from the $\mathrm{BP} \mathrm{T}_{1}$ state, is long-lived due to spin-forbidden charge recombination. The difference of the triplet photophysics of $\mathbf{1 X}$ in $\mathrm{H}_{2} \mathrm{O}$ vs. $\mathrm{MeOH}$, once again, can be rationalized by the different geometries. In the predominantly unfolded conformation in $\mathrm{MeOH}, \mathrm{CT}$ from the triplet state is very unlikely due to a large distance between the chromophore and the DNA bases (15 ̊). In the primarily stacked conformation in $\mathrm{H}_{2} \mathrm{O}$, CT becomes principally possible. The photochemistry of the $\mathrm{T}_{1}$ state that lies energetically lower than the $S_{1}$ state is decisive: $G$ and $A$ can clearly be photooxidized, whereas $\mathrm{C}$ and $\mathrm{T}$ are silent with respect to $\mathrm{CT}$. 


\section{Conclusion and outlook}

The central problem that currently emerges from experimental work on photoinduced charge transfer in DNA is that the conformational flexibility of double helical DNA is very complex and occurs on a wide range of time scales. In order to reduce this complexity we designed and synthesized four different dinucleotides $\mathbf{1 X}$ as models to study the conformational influence on photoinduced charge transfer in DNA. The major advantage of such small model compounds is that they are soluble both in water and $\mathrm{MeOH}$. The latter solvent was used to mimic the polarity in the interior of DNA. Using these dinucleotides the conformational influence on photoinduced singlet and triplet charge transfer was studied in full detail by both time-resolved transient absorption spectroscopy and theory. It turned out that the concept of controlling the conformational distribution by the solvent works well..$^{55}$ The dinucleotide $1 \mathrm{G}$ in $\mathrm{MeOH}$ adopts mainly an open and unfolded geometry, and in $\mathrm{H}_{2} \mathrm{O}$ primarily a stacked conformation. Charge transfer occurs only in the stacked conformation due to the significantly larger distance between BP and the nucleobase in the unfolded geometry. It became clear that the solvents not only control the conformational distribution, they actually gate the charge transfer due to differences in distance and degree of stacking. Therefore, our results give a full understanding of the photophysical properties of the singlet and triplet states of the BP chromophore in the context of each of the four different DNA bases. Since BP was chosen as the photoinduced electron acceptor the dinucleotides represent not only interesting biologically relevant models with respect to charge transfer and DNA damage but also the starting point for future applications in chemical biology, like photoaffinity labeling, and chemical photocatalysis.

\section{Abbreviations}

$\begin{array}{ll}\text { r.t. } & \text { Room temperature } \\ \text { ISC } & \text { Intersystem crossing } \\ \text { ET } & \text { Electron transfer } \\ \text { DADS } & \text { Decay associated difference spectra } \\ \text { SADS } & \text { Species associated difference spectra } \\ \text { TA } & \text { Transient absorption } \\ \text { NHE } & \text { Normal hydrogen electrode }\end{array}$

\section{Acknowledgements}

T.M., M.W., and M.W. thank the DFG research training group 1626 “Chemical Photocatalysis" gratefully for funding.

\section{Notes and references}

1 (a) Y. A. Berlin, I. V. Kurnikov, D. Baratan, M. A. Ratner and A. L. Burin, Top. Curr. Chem., 2004, 237, 1; (b) F. D. Lewis, H. Zhu, P. Daublain, T. Fiebig, M. Raytchev, Q. Wang and S. J. Vladimir, J. Am. Chem. Soc., 2005, 128, 791; (c) B. Giese, Bioorg. Med. Chem. Lett., 2006, 14, 6139; (d) T. Takada, K. Kawai, M. Fujitsuka and T. Majima, J. Am. Chem. Soc.,
2006, 128, 11012; (e) S. Kanvah, J. Joseph, G. B. Schuster, R. N. Barnett, C. L. Cleveland and U. Landman, Acc. Chem. Res., 2010, 43, 280; $(f)$ J. Genereux and J. K. Barton, Chem. Rev., 2010, 110, 1642.

2 F. C. Grozema, S. Tonzani, Y. A. Berlin, G. C. Schatz, L. D. A. Siebbeles and M. A. Ratner, J. Am. Chem. Soc., 2008, 130, 5157.

3 (a) M. A. O'Neill and J. K. Barton, J. Am. Chem. Soc., 2004, 126, 11471; (b) M. A. O'Neill and J. K. Barton, J. Am. Chem. Soc., 2004, 126, 13234.

4 P. Kaden, E. Mayer-Enthart, A. Trifonov, T. Fiebig and H.-A. Wagenknecht, Angew. Chem., Int. Ed., 2005, 44, 1636.

5 (a) F. D Lewis, A. K. Thazathveetil, T. A. Zeidan, J. Vura-Weis and M. R. Wasielewski, J. Am. Chem. Soc., 2010, 132, 444; (b) R. Carmieli, A. L. Semigh, S. M. M. Conron, A. K. Thazhaveetil, M. Fuki, Y. Kobori, F. D. Lewis and M. R. Wasieleswki, J. Am. Chem. Soc., 2012, 134, 11251.

6 (a) M. C. Cuquerella, V. Lhiaubet-Vallet, J. Cadet and M. A. Miranda, Acc. Chem. Res., 2012, 45, 1558; (b) B. Kohler, J. Phys. Chem. Lett., 2010, 1, 2047.

7 S. A. Fleming, Tetrahedron, 1995, 51, 12479.

8 G. Dorman and G. D. Prestwich, Biochemistry, 1994, 33, 5661.

9 R. E. Galardy, L. C. Craig, J. D. Jamieson and M. P. Printz, J. Biol. Chem., 1974, 249, 3510.

10 M. Fagnoni, D. Dondi, D. Ravelli and A. Albini, Chem. Rev., 2007, 107, 2725.

11 D. Ravelli, D. Dondi, M. Fagnoni and A. Albini, Chem. Soc. Rev., 2009, 38, 1999.

12 J. Svoboda and B. König, Chem. Rev., 2006, 106, 5413.

13 P. Wessig, Angew. Chem., Int. Ed., 2006, 45, 2168.

14 C. Müller, A. Bauer and T. Bach, Angew. Chem., Int. Ed., 2009, 48, 6640.

15 C. Müller, A. Bauer, M. M. Maturi, M. C. Cuquerella, M. A. Miranda and T. Bach, J. Am. Chem. Soc., 2011, 133, 16689.

16 D. F. Kauble, V. Lynch and M. J. Krische, J. Org. Chem., 2003, 68, 15.

17 A. Bauer, F. Westkamper, S. Grimme and T. Bach, Nature, 2005, 436, 1139.

18 T. E. Lehmann and A. Berkessel, J. Org. Chem., 1997, 62, 302.

19 T. E. Lehmann, G. Müller and A. Berkessel, J. Org. Chem., 2000, 65, 2508.

20 C. Paris, S. Encinas, N. Belmadoui, M. J. Climent and M. A. Miranda, Org. Lett., 2008, 10, 4409.

21 K. Musier-Forsyth and P. Schimmel, Biochemistry, 1994, 33, 773.

22 K. Nakatani, T. Yoshida and I. Saito, J. Am. Chem. Soc., 2002, 124, 2118.

23 K. Nakatani and I. Saito, Top. Curr. Chem., 2004, 236, 163. 24 W. Adam, M. A. Arnold, W. M. Nau, U. Pischel and C. R. Saha-Möller, J. Am. Chem. Soc., 2002, 124, 3893.

25 M. Weinberger and H.-A. Wagenknecht, Synthesis, 2012, 648. 26 T. Douki and J. Cadet, Int. J. Radiat. Biol., 1999, 75, 571.

27 B. Morin and J. Cadet, Photochem. Photobiol., 1994, 60, 102. 28 J. Perdew, Phys. Rev. B: Condens. Matter Mater. Phys., 1986, 33, 8822 . 
29 A. Schäfer, H. Horn and R. Ahlrichs, J. Chem. Phys., 1992, 97, 2571.

30 O. Christiansen, H. Koch and P. Jørgensen, Chem. Phys. Lett., 1995, 243, 409.

31 (a) T. Schwabe and S. Grimme, Phys. Chem. Chem. Phys., 2007, 9, 3397; (b) S. Grimme and M. Waletzke, J. Chem. Phys., 1999, 111, 5645.

32 A. D. Becke, J. Chem. Phys., 1993, 98, 5648.

33 F. Furche and R. Ahlrichs, J. Chem. Phys., 2002, 117, 7433.

34 C. Hättig and F. Weigend, J. Chem. Phys., 2000, 113, 5154.

35 C. Hättig and A. Kohn, J. Chem. Phys., 2002, 117, 6939.

36 C. Hättig, J. Chem. Phys., 2003, 118, 7751.

37 D. E. Woon and T. H. Dunning Jr., J. Chem. Phys., 1993, 98, 1358.

38 A. Dreuw, J. L. W. Worth and M. J. Head-Gordon, Chem. Phys., 2003, 119, 2943.

39 D. Tozer, J. Chem. Phys., 2003, 119, 12697.

40 S. Aloise, C. Ruckebusch, L. Blanchet, J. Réhault, G. Buntix and J.-P. Huvenne, J. Phys. Chem. A, 2008, 112, 224.

41 M. Wenninger, D. Fazio, U. Megerle, C. Trindler and S. S. Schiesser, ChemBioChem, 2011, 12, 703.

42 (a) F. Lewis, T. Wu, Y. Zang, R. Letsinger, S. Greenfield and M. Wasielewski, Science, 1997, 277, 673; (b) T. Fiebig, C. Wan and A. H. Zewail, ChemPhysChem, 2002, 3, 781.

43 U. Megerle, I. Pugliesi, C. Schriever, C. F. Sailer and E. Riedle, Appl. Phys. B: Lasers Opt., 2009, 96, 215.
44 R. V. Bensasson and J. C. D. Gramain, Faraday Trans. I, 1980, 76, 1801.

45 C. A. M. Seidel, A. Schulz and M. H. M. Sauer, J. Phys. Chem., 1996, 100, 5541.

46 K. R. Walczyk, G. S. Popkirov and R. N. Schindler, Ber. Bunsenges. Phys. Chem., 1995, 99, 1028.

47 (a) Y.-T. Kao, X. Guo, Y. Yang, Z. Liu, A. Hassanali, Q.-H. Song, L. Wang and D. Zhong, J. Am. Chem. Soc., 2012, 116, 9130; (b) J. Li, Z. Liu, C. Tan, X. Guo, L. Wang, A. Sancar and D. Zhong, Nature, 2010, 466, 887.

48 R. A. Marcus, J. Chem. Phys., 1956, 24, 966.

49 (a) D. Rehm and A. Weller, Isr. J. Chem., 1970, 8, 259; (b) V. Lhiaubet, N. Paillous and N. Chouini-Lalanne, Photochem. Photobiol., 2001, 74, 670; (c) N. A. Borisevich, D. V. Kazberuk, N. A. Lysak and G. B. Tolstotozhev, J. Appl. Spectrosc., 1994, 60, 193.

50 P. S. Rao and E. Hayon, J. Am. Chem. Soc., 1974, 96, 1295.

51 C. Reichardt, Solvent and Solvent Effects in Organic Chemistry, Wiley-VCH, Weinheim, 2003.

52 F. D. Lewis, R. S. Kalgutkar, Y. Wu, X. Liu, J. Liu, R. T. Hayes, S. E. Miller and M. R. Wasielewski, J. Am. Chem. Soc., 2000, 122, 12346.

53 J. W. Verhoeven, J. Photochem. Photobiol., C, 2006, 7, 40.

54 M. Weinberger, F. Berndt, R. Mahrwald, N. P. Ernsting and H.-A. Wagenknecht, J. Org. Chem., 2013, 78, 2589.

55 M. J. Lowe and J. A. Shellman, J. Mol. Biol., 1972, 65, 91. 